TRANSACTIONS OF THE

AMERICAN MATHEMATICAL SOCIETY

Volume 363, Number 1, January 2011, Pages 281-312

S 0002-9947(2010)05024-X

Article electronically published on August 23, 2010

\title{
THE MODULAR VARIETY OF HYPERELLIPTIC CURVES OF GENUS THREE
}

\author{
EBERHARD FREITAG AND RICCARDO SALVATI MANNI
}

\begin{abstract}
The modular variety of nonsingular and complete hyperelliptic curves with level-two structure of genus 3 is a 5-dimensional quasi-projective variety which admits several standard compactifications. The first one realizes this variety as a subvariety of the Siegel modular variety of level two and genus three.

It has 36 irreducible (isomorphic) components. One of the purposes of this paper will be to describe the equations of one of these components.

Two further models use the fact that hyperelliptic curves of genus three can be obtained as coverings of a projective line with 8 branch points. There are two important compactifications of this configuration space. The first one, $Y$, uses the semistable degenerated point configurations in $\left(P^{1}\right)^{8}$. This variety also can be identified with a Baily-Borel compactified ball-quotient $Y=$ $\overline{\mathcal{B}} / \Gamma[1-\mathrm{i}]$. We will describe these results in some detail and obtain new proofs including some finer results for them. The other compactification uses the fact that families of marked projective lines can degenerate to stable marked curves of genus 0 .

We use the standard notation $\bar{M}_{0,8}$ for this compactification. We have a
\end{abstract} diagram

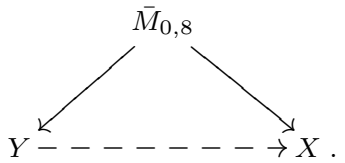

The horizontal arrow is only birational but not everywhere regular.

In this paper we find another realization of this triangle which uses the fact that there are graded algebras (closely related to algebras of modular forms) $A, B$ such that $X=\operatorname{proj}(A), Y=\operatorname{proj}(B)$.

\section{INTRODUCTION}

The modular variety of nonsingular and complete hyperelliptic curves with leveltwo structure of genus 3 is a 5 -dimensional quasi-projective variety which admits several standard compactifications. The first one comes from the period map, which realizes this variety as a subvariety of the Siegel modular variety of level two and genus three $\mathcal{H}_{3} / \Gamma_{3}[2]$. We denote the hyperelliptic locus within $\mathcal{H}_{3} / \Gamma_{3}[2]$ by $\mathcal{I}_{3}[2]$ and its closure in the Satake compactification by

$$
\overline{\mathcal{I}_{3}[2]} \subset \overline{\mathcal{H}_{3} / \Gamma_{3}[2]} .
$$

Received by the editors December 2, 2007 and, in revised form, January 29, 2009.

2010 Mathematics Subject Classification. Primary 11F46, 11F55.

(C)2010 American Mathematical Society 
The hyperelliptic locus has 36 irreducible (isomorphic) components, which correspond to the 36 even theta characteristics. We denote the component of the characteristic $m$ by

$$
X={\overline{\mathcal{I}_{3}[2]}}^{m} .
$$

One of the purposes of this paper will be to describe the equations of $X$ in a suitable projective embedding and its Hilbert function (5.8). It will turn out that $X$ is normal.

Two further models use the fact that hyperelliptic curves of genus three can be obtained as coverings of a projective line with 8 branch points. The level-two structure of the curve corresponds to an ordering of these 8 points. This leads to the configuration space of 8 ordered points on a line. There are two important compactifications of this configuration space. The first one comes from geometric invariant theory using so-called semistable degenerate point configurations in $\left(P^{1}\right)^{8}$. We denote this GIT-compactification by $Y$. The equations of this variety in a suitable projective embedding (by so-called Specht polynomials) are known. For a general approach to the GIT-model $\left(P^{1}\right)^{n} / / \mathrm{SL}(2)$ we refer to the papers HMSV1, HMSV2. In the special case $n=8$, this variety can also be identified with a Baily-Borel compactified ball-quotient Ko2

$$
Y=\overline{\mathcal{B} / \Gamma[1-\mathrm{i}]} .
$$

We will describe these results in some detail and obtain new proofs including some finer results for them. We will see for example that the graded algebra describing $\left(P^{1}\right)^{8} / / \mathrm{SL}(2)$ is a Gorenstein ring (Theorem 1.5) and that it describes the full ring of modular forms of $\Gamma[1-\mathrm{i}]$ (Theorem 2.4). This part is essentially due to Kondo Ko2, but we can avoid the theory of $K 3$-surfaces, which is an essential part of Kondo's approach.

The other compactification uses the fact that families of marked projective lines $\left(P^{1}, x_{1}, \ldots, x_{8}\right)$ can degenerate to stable marked curves $\left(C, x_{1}, \ldots, x_{8}\right)$ of genus 0 .

We use the standard notation $\bar{M}_{0,8}$ for this compactification. It is known that $\bar{M}_{0, n}$ for arbitrary $n$ is a smooth and projective variety.

In $\mathrm{Ka}$, (see also $\mathrm{AL}$ ) it has been proved that the birational map

$$
\bar{M}_{0, n} \longrightarrow\left(P^{1}\right)^{n} / / \mathrm{SL}(2)
$$

is everywhere regular. It is also known that there is natural everywhere regular map (Torelli map)

$$
\bar{M}_{0, n} \longrightarrow \overline{\mathcal{H}_{g} / \Gamma_{g}[2]} \quad \text { (Satake compactification) }
$$

with $n=2 g+2$. Hence in the case $n=8$ we have a diagram

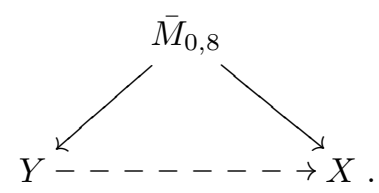

The horizontal arrow is only birational but not everywhere regular. 
In this paper we find another realization of this triangle which uses the fact that there are graded algebras (closely related to algebras of modular forms) $A, B$ such that

$$
X=\operatorname{proj}(A), \quad Y=\operatorname{proj}(B) .
$$

The rational map $Y--\rightarrow X$ is induced by a homomorphism of graded algebras

$$
A \longrightarrow B
$$

This homomorphism rests on the theory of Thomae (19th century), in which the thetanullwerte of hyperelliptic curves have been computed. We use Mumford's approach $[\mathrm{Mu}$ for the construction of this homomorphism. Using the explicit equations for $A, B$ we can compute the base locus of the rational map $Y--\rightarrow X$. It turns out to be the union of $56 P^{3}$ (Proposition 6.2). Blowing up the base locus we get a projective variety $Y^{*}$ and a diagram

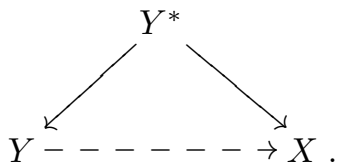

But the model $Y^{*}$ is singular. The space $Y$ contains 35 special points (corresponding to nonstable points in the GIT-model or to the cusps in the ball-quotient model). Their inverse images in $Y^{*}$ are 35 disjoint rational surfaces. Blowing up them we get a dominant smooth model and a diagram

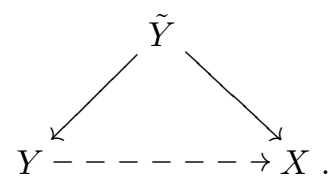

We will see that $\tilde{Y}$ and $\bar{M}_{0,8}$ are isomorphic; see Theorem 6.7. This can be considered as an explicit description of $\bar{M}_{0,8}$.

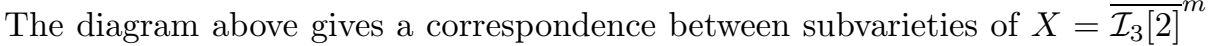
and of $Y=\overline{\mathcal{B} / \Gamma[1-\mathrm{i}]}$. (Take the inverse image of a subvariety of $X$ in $\tilde{Y}$ and project to $Y$ and conversely.) This correspondence explains several combinatorial similarities between the two models. These similarities can be described best if one uses the ball model to describe $Y$. The reason is that in the description of the Siegel and the ball models, the space $\mathbb{F}_{2}^{6}$ occurs. In the Siegel case it occurs as a symplectic space; its elements are so-called characteristics. In the ball case it occurs as a quadratic space. The symmetry of both models can be explained by the fact that in characteristic 2 the symplectic and orthogonal world come together. This will be described in the last section.

The second named author is grateful to Corrado De Concini and Shigeyuki Kondo for helpful and enlightening discussions. He would like also to thank Herbert Lange and Hanspeter Kraft for bringing his attention to the papers $\mathrm{AL}$ and $[\mathrm{HO}]$. 


\section{The CONFIgurAtion SPACE FOR EIGHT POINTS ON A LINE}

We recall some basic facts about the configuration space of eight points on the line. With the help of computer computations we get very easy proofs of them and also some new insight.

We consider the subset

$$
\mathcal{X}(8) \subset P^{1}(\mathbb{C})^{8}
$$

of ordered sets of 8 points on $P^{1}$. The elements of $P^{1}(\mathbb{C})$ can be represented by columns $\left(\begin{array}{l}a \\ b\end{array}\right)$, which are different from zero and which are defined up to a constant factor. Hence the elements of $\mathcal{X}(8)$ can be represented by matrices

$$
M:=\left(\begin{array}{llll}
a_{1} & a_{2} & \ldots & a_{8} \\
b_{1} & b_{2} & \ldots & b_{8}
\end{array}\right)
$$

Since $\mathrm{SL}(2, \mathbb{C})$ acts on $P^{1}(\mathbb{C})$, we get an action of $\mathrm{SL}(2, \mathbb{C})$ on $\mathcal{X}(8)$ by multiplication from the left. The configuration space is defined as

$$
X(8)=\mathrm{SL}(2, \mathbb{C}) \backslash \mathcal{X}(8)
$$

We recall that a tableau is a matrix

$$
\left(\begin{array}{c}
i_{1} \ldots i_{4} \\
j_{1} \ldots j_{4}
\end{array}\right)
$$

which contains as entries all digits $i, 1 \leq i \leq 8$, and with the property

$$
i_{1}<i_{2}<i_{3}<i_{4}, \quad i_{1}<j_{1}, \quad i_{2}<j_{2}, \quad i_{3}<j_{3}, \quad i_{4}<j_{4}
$$

There are 105 tableaux. The tableau is called standard if, in addition, $j_{1}<j_{2}<$ $j_{3}<j_{4}$. There are 14 standard tableaux:

$$
\begin{aligned}
& \left(\begin{array}{llll}
1 & 2 & 3 & 4 \\
5 & 6 & 7 & 8
\end{array}\right) \quad\left(\begin{array}{llll}
1 & 2 & 3 & 5 \\
4 & 6 & 7 & 8
\end{array}\right) \quad\left(\begin{array}{llll}
1 & 2 & 3 & 6 \\
4 & 5 & 7 & 8
\end{array}\right) \quad\left(\begin{array}{llll}
1 & 2 & 3 & 7 \\
4 & 5 & 6 & 8
\end{array}\right) \\
& \left(\begin{array}{llll}
1 & 2 & 4 & 5 \\
3 & 6 & 7 & 8
\end{array}\right) \quad\left(\begin{array}{llll}
1 & 2 & 4 & 6 \\
3 & 5 & 7 & 8
\end{array}\right) \quad\left(\begin{array}{llll}
1 & 2 & 4 & 7 \\
3 & 5 & 6 & 8
\end{array}\right) \quad\left(\begin{array}{llll}
1 & 2 & 5 & 6 \\
3 & 4 & 7 & 8
\end{array}\right) \\
& \left(\begin{array}{llll}
1 & 2 & 5 & 7 \\
3 & 4 & 6 & 8
\end{array}\right) \quad\left(\begin{array}{llll}
1 & 3 & 4 & 5 \\
2 & 6 & 7 & 8
\end{array}\right) \quad\left(\begin{array}{llll}
1 & 3 & 4 & 6 \\
2 & 5 & 7 & 8
\end{array}\right) \quad\left(\begin{array}{llll}
1 & 3 & 4 & 7 \\
2 & 5 & 6 & 8
\end{array}\right) \\
& \left(\begin{array}{llll}
1 & 3 & 5 & 6 \\
2 & 4 & 7 & 8
\end{array}\right) \quad\left(\begin{array}{llll}
1 & 3 & 5 & 7 \\
2 & 4 & 6 & 8
\end{array}\right)
\end{aligned}
$$

To each tableau one associates the expression

$$
D(M)=\prod_{\nu=1}^{4}\left(a_{i_{\nu}} b_{j_{\nu}}-a_{j_{\nu}} b_{i_{\nu}}\right)
$$

This leads to the so-called Specht polynomial

$$
D\left(\begin{array}{ccc}
1 & \cdots & 1 \\
X_{1} & \cdots & X_{8}
\end{array}\right)=\left(X_{i_{1}}-X_{j_{1}}\right)\left(X_{i_{2}}-X_{j_{2}}\right)\left(X_{i_{3}}-X_{j_{3}}\right)\left(X_{i_{4}}-X_{j_{4}}\right)
$$

The space generated by all 105 Specht polynomials has dimension 14. A basis is given by the Specht polynomials of the standard tableaux [Koi]. We denote them 
by $Y_{1}(X), \ldots, Y_{14}(X)$ in the above ordering:

$$
\begin{gathered}
Y_{1}=\left(X_{1}-X_{5}\right)\left(X_{2}-X_{6}\right)\left(X_{3}-X_{7}\right)\left(X_{4}-X_{8}\right) \\
Y_{2}=\left(X_{1}-X_{4}\right)\left(X_{2}-X_{6}\right)\left(X_{3}-X_{7}\right)\left(X_{5}-X_{8}\right) \\
Y_{3}=\left(X_{1}-X_{4}\right)\left(X_{2}-X_{5}\right)\left(X_{3}-X_{7}\right)\left(X_{6}-X_{8}\right) \\
Y_{4}=\left(X_{1}-X_{4}\right)\left(X_{2}-X_{5}\right)\left(X_{3}-X_{6}\right)\left(X_{7}-X_{8}\right) \\
Y_{5}=\left(X_{1}-X_{3}\right)\left(X_{2}-X_{6}\right)\left(X_{4}-X_{7}\right)\left(X_{5}-X_{8}\right) \\
Y_{6}=\left(X_{1}-X_{3}\right)\left(X_{2}-X_{5}\right)\left(X_{4}-X_{7}\right)\left(X_{6}-X_{8}\right) \\
Y_{7}=\left(X_{1}-X_{3}\right)\left(X_{2}-X_{5}\right)\left(X_{4}-X_{6}\right)\left(X_{7}-X_{8}\right) \\
Y_{8}=\left(X_{1}-X_{3}\right)\left(X_{2}-X_{4}\right)\left(X_{5}-X_{7}\right)\left(X_{6}-X_{8}\right) \\
Y_{9}=\left(X_{1}-X_{3}\right)\left(X_{2}-X_{4}\right)\left(X_{5}-X_{6}\right)\left(X_{7}-X_{8}\right) \\
Y_{10}=\left(X_{1}-X_{2}\right)\left(X_{3}-X_{6}\right)\left(X_{4}-X_{7}\right)\left(X_{5}-X_{8}\right) \\
Y_{11}=\left(X_{1}-X_{2}\right)\left(X_{3}-X_{5}\right)\left(X_{4}-X_{7}\right)\left(X_{6}-X_{8}\right) \\
Y_{12}=\left(X_{1}-X_{2}\right)\left(X_{3}-X_{5}\right)\left(X_{4}-X_{6}\right)\left(X_{7}-X_{8}\right) \\
Y_{13}=\left(X_{1}-X_{2}\right)\left(X_{3}-X_{4}\right)\left(X_{5}-X_{7}\right)\left(X_{6}-X_{8}\right) \\
Y_{14}=\left(X_{1}-X_{2}\right)\left(X_{3}-X_{4}\right)\left(X_{5}-X_{6}\right)\left(X_{7}-X_{8}\right) .
\end{gathered}
$$

It is clear that these polynomials define a holomorphic map

$$
X(8) \longrightarrow P^{13}(\mathbb{C}) .
$$

It is easy to check and well known that $X(8) \hookrightarrow P^{13}$ is a smooth embedding Koi]. We denote by $\bar{X}(8)$ the closure of the image of $X(8)$ in $P^{13}(\mathbb{C})$. Hence

$$
\bar{X}(8)=\operatorname{proj} \mathbb{C}\left[Y_{1}, \ldots, Y_{14}\right] / \mathcal{J}
$$

where $\mathcal{J}$ is the ideal of relations between the Specht polynomials $Y_{i}(X)$. The ideal $\mathcal{J}$ can be computed by elimination: Consider the polynomial ring in 28 variables $\mathbb{C}\left[X_{1}, \ldots, X_{14}, T_{1}, \ldots, T_{14}\right]$ and the ideal generated by the relations $T_{i}-Y_{i}(1 \leq i \leq$ 14). Then $\mathcal{J}$ is the intersection of this ideal with $\mathbb{C}\left[Y_{1}, \ldots, Y_{14}\right]$. With computer algebra one can verify now 1 a slightly modified result of Koike [Koi]:

1.1. Lemma (Koike). The ideal $\mathcal{J}$ is generated by the polynomials

$$
\begin{aligned}
J_{1} & =Y_{9} Y_{13}-Y_{8} Y_{14}, \\
J_{2} & =Y_{2} Y_{12}-Y_{3} Y_{12}-Y_{5} Y_{12}+Y_{6} Y_{12}-Y_{1} Y_{14}+Y_{3} Y_{14}+Y_{5} Y_{14}-Y_{7} Y_{14} \\
& -Y_{8} Y_{14}+Y_{9} Y_{14}-Y_{11} Y_{14}+Y_{13} Y_{14}, \\
J_{3} & =Y_{7} Y_{11}-Y_{6} Y_{12}, \\
J_{4} & =Y_{4} Y_{11}-Y_{3} Y_{12}-Y_{4} Y_{13}+Y_{3} Y_{14}, \\
J_{5} & =Y_{2} Y_{11}-Y_{5} Y_{11}-Y_{3} Y_{12}+Y_{6} Y_{12}-Y_{1} Y_{13}+Y_{5} Y_{13}-Y_{7} Y_{13}+Y_{3} Y_{14} \\
& -Y_{11} Y_{14}+Y_{13} Y_{14}, \\
J_{6} & =Y_{7} Y_{10}-Y_{9} Y_{10}-Y_{5} Y_{12}+Y_{5} Y_{14}, \\
J_{7} & =Y_{6} Y_{10}-Y_{8} Y_{10}-Y_{5} Y_{11}+Y_{8} Y_{11}-Y_{9} Y_{11}+Y_{5} Y_{13}-Y_{6} Y_{13}+Y_{6} Y_{14}, \\
J_{8} & =Y_{4} Y_{10}-Y_{9} Y_{10}-Y_{3} Y_{12}-Y_{5} Y_{12}+Y_{6} Y_{12}-Y_{4} Y_{13}-Y_{1} Y_{14} \\
& +Y_{3} Y_{14}+Y_{4} Y_{14}+Y_{5} Y_{14}-Y_{7} Y_{14}+Y_{10} Y_{14}-Y_{11} Y_{14}+Y_{14}, \\
J_{9} & =Y_{3} Y_{10}-Y_{8} Y_{10}-Y_{5} Y_{11}+Y_{8} Y_{11}-Y_{9} Y_{11}-Y_{3} Y_{12}+Y_{6} Y_{12}-Y_{1} Y_{13} \\
& +Y_{5} Y_{13}-Y_{7} Y_{13}+Y_{10} Y_{13}-Y_{11} Y_{13}+Y_{3} Y_{14}+Y_{13} Y_{14}, \\
J_{10} & =Y_{2} Y_{7}-Y_{3} Y_{7}-Y_{1} Y_{9}+Y_{3} Y_{9}-Y_{9} Y_{11}-Y_{5} Y_{12}+Y_{6} Y_{12}+Y_{5} Y_{14} \\
& -Y_{7} Y_{14}+Y_{9} Y_{14},
\end{aligned}
$$

\footnotetext{
${ }^{1}$ The computer algebra calculation has to be done over the field $\mathbb{Q}$ instead $\mathbb{C}$, which is sufficient.
} 


$$
\begin{aligned}
& J_{11}=Y_{4} Y_{6}-Y_{3} Y_{7}-Y_{4} Y_{8}+Y_{7} Y_{8}+Y_{3} Y_{9}-Y_{6} Y_{9}-Y_{7} Y_{13}+Y_{6} Y_{14}, \\
& J_{12}=Y_{2} Y_{6}-Y_{3} Y_{7}-Y_{1} Y_{8}+Y_{3} Y_{9}-Y_{5} Y_{11}-Y_{9} Y_{11}+Y_{6} Y_{12}+Y_{5} Y_{13} \\
& \quad-Y_{7} Y_{13}+Y_{8} Y_{14} \\
& J_{13}=Y_{4} Y_{5}-Y_{3} Y_{7}-Y_{4} Y_{8}+Y_{7} Y_{8}-Y_{1} Y_{9}+Y_{3} Y_{9}+Y_{4} Y_{9}-Y_{7} Y_{9}-Y_{9} Y_{11} \\
& \quad-Y_{5} Y_{12}+Y_{6} Y_{12}-Y_{7} Y_{13}+Y_{5} Y_{14}+Y_{9} Y_{14}, \\
& J_{14}=Y_{3} Y_{5}-Y_{3} Y_{7}-Y_{1} Y_{8}+Y_{3} Y_{9}-Y_{5} Y_{11}-Y_{9} Y_{11}+Y_{6} Y_{12}+Y_{5} Y_{13} \\
& \quad-Y_{7} Y_{13}+Y_{8} Y_{14} .
\end{aligned}
$$

We want to describe the boundary $\bar{X}(8)-X(8)$. Koike compares $\bar{X}(8)$ with a certain ball quotient. This relies on a paper of Kondo who identifies the Satake compactified ball quotient with the GIT-compactification of $X(8)$. Since Kondo's argument is not completely correct (see section 2), we give here in some detail a self-contained complete description of $\bar{X}(8)$. We need some results from geometric invariant theory which gives the so-called GIT-compactification of $X(8)$.

Let $R$ be the graded algebra $R=\sum_{n=0}^{\infty} R_{n}$, where $R_{n}$ denotes the space of all polynomials in the entries of a $2 \times 8$ matrix $M$ which are homogeneous of the same degree $n$ in each column of $M$. Hence $\operatorname{proj}(R)=P^{1}(\mathbb{C})^{8}$. There is a natural action of the group $G=\mathrm{SL}(2, \mathbb{C})$ on $R$. The ring of invariants $R^{G}$ is finitely generated. One defines

$$
\bar{X}(8)^{\mathrm{GIT}}:=\operatorname{proj}\left(R^{G}\right) .
$$

Recall that a point from $P^{1}(\mathbb{C})^{8}$ is called semistable if there is some element from $R_{n}^{G}$ which doesn't vanish on it. The set of all semistable points $P^{1}(\mathbb{C})_{\mathrm{ss}}^{8} \subset P^{1}(\mathbb{C})^{8}$ is a $G$-invariant Zariski open subset. There is an obvious map $P^{1}(\mathbb{C})_{\mathrm{ss}}^{8} \rightarrow \bar{X}(8)^{\mathrm{GIT}}$, and this is a categorical quotient. Since we are in a geometric situation, this map is surjective.

It is known that a point in $P^{1}(\mathbb{C})^{8}$ is semistable if and only if not more than 4 of its coordinates are the same. It follows that there does not exist a common zero for all Specht polynomials at once. Hence we have a map $P^{1}(\mathbb{C})_{\mathrm{ss}}^{8} \rightarrow \bar{X}(8)$, and from the universal property we get a map

$$
\bar{X}(8)^{\mathrm{GIT}} \longrightarrow \bar{X}(8) .
$$

We will see that this is a biholomorphic map. This actually follows from a known theorem from Kempe; see HMSV1, HMSV2:

1.2. Proposition. The ring $R^{G}$ is generated by its elements of degree one. (These are the Specht polynomials.)

We don't need to use this theorem. We will obtain it as a consequence of a sharper result which seems to be new (2).

First of all one sees from the description of the semistable points that the irreducible subvarieties of dimension 4 of the boundary $\bar{X}(8)^{\mathrm{GIT}}-X(8)$ are defined by the subsets of $P^{1}(\mathbb{C})^{8}$ where two points agree. Hence these irreducible subsets perform one orbit under the group $S_{8}$. It follows that the boundary $\bar{X}(8)-X(8)$ also contains at most one orbit of irreducible subvarieties of dimension 4 . It is really one orbit, since one can check easily by means of computer algebra that the subvariety defined by $J_{9}=J_{10}=J_{11}=J_{12}=J_{13}=J_{14}=0$ is of dimension 4 . It 
is easy to exhibit a special point in this subvariety which is smooth in the whole $\bar{X}(8)$. Hence we see:

1.3. Lemma. The ring $\mathbb{C}\left[Y_{1}, \ldots, Y_{14}\right] / \mathcal{J}$ is regular in codimension one.

Another calculation with computer algebra gives an explicit minimal resolution of this ring. It turns out that the resolution is symmetric, which implies that the ring is a Cohen-Macaulay ring:

1.4. Lemma. Let $A$ be the polynomial ring $\mathbb{C}\left[Y_{1}, \ldots, Y_{14}\right]$. The minimal resolution of the ring $B:=\mathbb{C}\left[Y_{1}, \ldots, Y_{14}\right] / \mathcal{J}$ is of the form

$$
\begin{aligned}
0 \rightarrow A^{1} \rightarrow A^{14} & \rightarrow A^{175} \rightarrow A^{512} \rightarrow A^{700} \\
& \rightarrow A^{512} \rightarrow A^{175} \rightarrow A^{14} \rightarrow A^{1} \rightarrow B \rightarrow 0 .
\end{aligned}
$$

Corollary. The ring $B$ is a Cohen-Macaulay ring; even more, it is a Gorenstein ring.

Using Serre's criterion for normality we now get:

1.5. Theorem. The ring $\mathbb{C}\left[Y_{1}, \ldots, Y_{14}\right] / \mathcal{J}$ is a normal Gorenstein ring.

1. Corollary (Kempe). This ring agrees with the full ring of invariants $R^{G}$, which hence is generated by the elements of lowest degree (hence by the Specht polynomials).

2. Corollary (Kempe). The map

$$
\bar{X}(8)^{G I T} \stackrel{\sim}{\longrightarrow} \bar{X}(8)
$$

is biholomorphic.

We want to point out that Kempe's result has been generalized in HMSV1 and HMSV2 to point configurations of an arbitrary number of points in the projective line.

From the computation of the resolution one can also read off:

1.6. Theorem. The Hilbert function of $R^{G} \cong \mathbb{C}\left[Y_{1}, \ldots, Y_{14}\right] / \mathcal{J}$ is

$$
\begin{aligned}
\sum_{n=0}^{\infty} \operatorname{dim} R_{n}^{G} t^{n} & =\frac{1+8 t+22 t^{2}+8 t^{3}+t^{4}}{(1-t)^{6}} \\
& =1+14 t+91 t^{2}+364 t^{3}+1085 t^{4}+5719 t^{6}+\cdots
\end{aligned}
$$

This formula is in concordance with other formulae in the literature. Howe [Ho] for example gives

$$
\operatorname{dim} R_{n}^{G}=\frac{n^{5}+5 n^{4}+11 n^{3}+13 n^{2}+9 n+3}{3},
$$

which is the same as an easy computation shows. The fact that Howe's formula is true for all $n$ reflects that $R^{G}$ is Gorenstein.

Another remarkable formula, communicated to us by De Concini, is a consequence of the results obtained in $\mathrm{DP}$ and is based on counting the standard 
tableaux:

$$
\begin{aligned}
\operatorname{dim} R_{n}^{G}= & \sum_{i_{1}+2 i_{2}+\cdots+n i_{n}=4 n}\left(\begin{array}{c}
8 \\
i_{1}
\end{array}\right)\left(\begin{array}{c}
8-i_{1} \\
i_{2}
\end{array}\right) \cdots\left(\begin{array}{c}
8-i_{1}-\cdots-i_{n-1} \\
i_{n}
\end{array}\right) \\
& -\sum_{i_{1}+2 i_{2}+\cdots+n i_{n}=4 n-1}\left(\begin{array}{c}
8 \\
i_{1}
\end{array}\right)\left(\begin{array}{c}
8-i_{1} \\
i_{2}
\end{array}\right) \cdots\left(\begin{array}{c}
8-i_{1}-\cdots-i_{n-1} \\
i_{n}
\end{array}\right) .
\end{aligned}
$$

\section{A BAll QUotient}

The ring $\mathbb{C}\left[Y_{1}, \ldots, Y_{14}\right] / \mathcal{J}$ of the previous section turns out to be the ring of modular forms on a certain ball quotient. This is related to the fact that $\bar{X}(8)$ is isomorphic to a Baily-Borel compactified ball quotient. These results are essentially due to Kondo Ko2. Here we amend and extend some of his arguments.

The configuration space of 8 points on a line is related to the even lattice

$$
L:=U \oplus U(2) \oplus D_{4}(-1) \oplus D_{4}(-1) .
$$

Here as usual $U=\mathbb{Z}^{2}$ with the quadratic form $x_{1} x_{2}$ and

$$
D_{4}=\left\{\left(x_{1}, x_{2}, x_{3}, x_{4}\right) \in \mathbb{Z}^{4} ; \quad x_{1}+\cdots+x_{4} \equiv 0 \quad \bmod 2\right\}, \quad q(x)=x_{1}^{2}+\cdots+x_{4}^{2} .
$$

The notation $M(m)$ means that one takes the same abelian group $M$, but the quadratic form is multiplied by $m$. Hence $\mathrm{U} \oplus \mathrm{U}(2)=\mathbb{Z}$ with the quadratic form $x_{1} x_{2}+2 x_{3} x_{4}$. We denote by $L^{\prime}$ the dual lattice of $L$. The discriminant group $L^{\prime} / L$ is isomorphic to $\mathbb{F}_{2}^{6}$. The isomorphism can be chosen such that the diagram

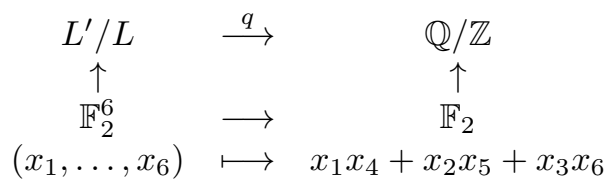

commutes. Here $\mathbb{F}_{2}$ is embedded into $\mathbb{Q} / \mathbb{Z}$ by sending $0 \mapsto 0$ and $1 \mapsto 1 / 2$. It will be basic for us that the orthogonal group (so-called even type) $\mathrm{O}\left(\mathbb{F}_{2}^{6}\right)$ is isomorphic to the symmetric group $S_{8}$. We choose an isomorphism

$$
S_{8} \stackrel{\sim}{\longrightarrow} \mathrm{O}\left(\mathbb{F}_{2}^{6}\right) \text {. }
$$

A natural concrete construction of such an isomorphism will be given in section 4 . At the moment the choice is not important.

We now describe a certain graded algebra, which is related to $\mathrm{O}\left(\mathbb{F}_{2}^{6}\right)$. This is the third member of a sequence of algebras related to $\mathrm{O}\left(\mathbb{F}_{2}^{2 m}\right)[\mathrm{FS}$. We recall very briefly its definition. We attach to each maximal totally isotropic subspace $I \subset \mathbb{F}_{2}^{2 m}$ (of dimension $m$ ) a variable $X_{I}$ and we consider the subring

$$
\mathcal{R}_{m}:=\mathbb{C}\left[\ldots X_{I}-X_{J} \ldots\right]
$$

of the polynomial ring $\mathbb{C}\left[\ldots X_{I} \ldots\right]$ in all these variables. We define an ideal $\mathcal{I}_{m} \subset$ $\mathcal{R}_{m}$. It is the sum of a linear part and a quadratic part,

$$
\mathcal{I}_{m}=\mathcal{I}_{m}^{\text {lin }}+\mathcal{I}_{m}^{\text {qua }} .
$$

We first define the linear part. For this we need the characteristic function

$$
\chi_{I}: \mathbb{F}_{2}^{2 m} \longrightarrow \mathbb{C}
$$


of a maximal totally isotropic subspace. Let $A \subset \mathbb{F}_{2}^{2 m}$ be a totally isotropic subspace of dimension $m-2$. There are 6 maximal totally isotropic subspaces $I_{1}, \ldots, I_{6}$ containing $A$. Their ordering can be chosen such that

$$
\left(\chi_{I_{1}}-\chi_{I_{2}}\right)+\left(\chi_{I_{3}}-\chi_{I_{4}}\right)+\left(\chi_{I_{5}}-\chi_{I_{6}}\right)=0 .
$$

The linear form

$$
\left(X_{I_{1}}-X_{I_{2}}\right)+\left(X_{I_{3}}-X_{I_{4}}\right)+\left(X_{I_{5}}-X_{I_{6}}\right)
$$

is determined by $A$ up to a sign. The linear part $\mathcal{I}_{m}^{\text {lin }}$ is generated by all linear forms, where $A$ runs through all the spaces $A$. Next we define the quadratic part. Again we consider a totally isotropic subspace $A \subset \mathbb{F}_{2}^{2 m}$ of dimension $m-2$ and the 6 maximal totally isotropic subspaces $I_{1}, \ldots, I_{6}$ containing it. It is easy to check that the numbering can be chosen such that the relation

$$
\left(\chi_{I_{1}}-\chi_{I_{2}}\right)\left(\chi_{I_{1}}-\chi_{I_{4}}\right)=\left(\chi_{I_{3}}-\chi_{I_{6}}\right)\left(\chi_{I_{5}}-\chi_{I_{6}}\right)
$$

holds. Again the polynomial

$$
\left(X_{I_{1}}-X_{I_{2}}\right)\left(X_{I_{1}}-X_{I_{4}}\right)-\left(X_{I_{3}}-X_{I_{6}}\right)\left(X_{I_{5}}-X_{I_{6}}\right)
$$

is determined by $A$ up to the sign, and we can consider the ideal $\mathcal{I}_{m}^{\text {qua }}$, which is generated by all these quadratic polynomials.

The ring $\mathcal{R}_{m} / \mathcal{I}_{m}$, which we defined so far in a formal way, seems to be very interesting. For example the cases $m=5$ and $m=6$ are related to Enriques surfaces. Here we are interested in the case $m=3$.

First of all one computes that a linear combination $\sum_{V} C_{V} X_{V}$ is in the ideal $\mathcal{I}_{3}$ if and only if $\sum_{V} C_{V} \chi_{V}=0$. The dimension of the space generated by all $\chi_{V}-\chi_{W}$ can be computed and it is 14 . Hence $\mathcal{R}_{3} / \mathcal{I}_{3}^{\text {lin }}$ is a polynomial ring in 14 variables. The group $\mathrm{O}\left(\mathbb{F}_{2}^{6}\right)$ acts on this ring. The action comes from an irreducible linear representation on the lowest degree part. Since the subgroup of index two $A_{8} \subset S_{8}$ admits only one irreducible representation of dimension 14, we obtain an essentially unique $A_{8}$-equivariant isomorphism

$$
\mathcal{R}_{3} / \mathcal{I}_{3}^{\operatorname{lin}} \longrightarrow \mathbb{C}\left[Y_{1}, \ldots, Y_{14}\right]
$$

where on the right hand side we have the ring described in section one. It turns out to be $\mathrm{O}\left(\mathbb{F}_{2}^{6}\right)=S_{8}$-equivariant, and a direct computation shows that the quadratic relations go to 0 . (This can be considered as a justification for the introduction of the ideal $\mathcal{I}_{n}$.) Hence we get a homomorphism

$$
\mathcal{R}_{3} / \mathcal{I}_{3} \longrightarrow \mathbb{C}\left[Y_{1}, \ldots, Y_{14}\right] / \mathcal{J} \text {. }
$$

It is no problem to check by a concrete calculation:

2.1. Theorem. The homomorphism

$$
\mathcal{R}_{3} / \mathcal{I}_{3} \longrightarrow \mathbb{C}\left[Y_{1}, \ldots, Y_{14}\right] / \mathcal{J}
$$

is an $\mathrm{O}\left(\mathbb{F}_{2}^{6}\right) \cong S_{8}$-equivariant isomorphism.

Corollary. The ring $\mathcal{R}_{3} / \mathcal{I}_{3}$ is a normal ring of Krull dimension 5.

We want to mention that the Krull dimension of $\mathcal{R}_{m} / \mathcal{I}_{m}$ is unknown for $m>3$.

The ring $\mathcal{R}_{3} / \mathcal{I}_{3}$ is related to modular forms $[\mathrm{FS}$. There is a subgroup of index two of $\mathrm{O}\left(L \otimes_{\mathbb{G}} \mathbb{R}\right)$, which doesn't contain the reflections along vectors of positive norm. Its intersection with $\mathrm{O}(L)$ is denoted by $\mathrm{O}^{\prime}(L)$. The discriminant kernel is

$$
\Gamma_{L}=\operatorname{kernel}\left(\mathrm{O}^{\prime}(L) \longrightarrow \mathrm{O}\left(L^{\prime} / L\right)\right) \text {. }
$$


The Borcherds additive lifting attaches to each maximal totally isotropic subspace a modular form [FS. This gives a homomorphism

$$
\mathcal{R}_{3} / \mathcal{I}_{3}^{\operatorname{lin}} \longrightarrow A\left(\Gamma_{L}\right),
$$

where $A\left(\Gamma_{L}\right)$ denotes the ring of modular forms with respect to $\Gamma_{L}$. It is possible to derive certain quadratic relations $[\mathrm{FS}$. Kondo derived quartic relations, but Koike Koi pointed out that they are consequences of quadratic relations. These relations can be explained naturally by means of the theory of Borcherds products. To explain this, we have to recall the notion of a star [FS].

2.2. Definition. A star $S$ is a set of 4 anisotropic vectors in $\mathbb{F}^{6}$, which is a coset of a 2-dimensional totally isotropic subspace.

(Kondo considers instead of stars "maximal totally singular subspaces". They are in one-to-one correspondence with the stars. The set of anisotropic elements of such a space is a star, and this gives a one-to-one correspondence between stars and maximal totally singular subspaces.) To every star a certain Borcherds product can be associated. To explain this we have to recall how to associate to an element $\alpha \in \mathbb{F}_{2}^{6}$ of nonzero norm a certain Heegner divisor $\mathcal{H}_{\alpha}$ on the symmetric domain which is associated to $\mathrm{O}(L)$. Recall that the elements of this symmetric domain can be considered as two-dimensional positive definite subspaces of $L \otimes_{\mathbb{G}} \mathbb{R}$. Then $\mathcal{H}_{\alpha}$ consists of all such subspaces, which are orthogonal to some

$$
\delta \in L^{*}, \quad(\delta, \delta)=-1, \quad \delta \text { over } \alpha .
$$

If $S$ is star, we denote by $\mathcal{H}_{S}$ the union of the four $\mathcal{H}_{\alpha}, \alpha \in S$. The basic fact is that the additive lift space contains for every star $S$ a form $f_{S}$, whose zero divisor contains $\mathcal{H}_{S}$. Since there is also a Borcherds product of the same weight with this property, the zero divisor equals $\mathcal{H}_{S}$. From this it is possible to derive directly quadratic relations [FS] and to prove:

\subsection{Lemma. The Borcherds additive lifting gives a homomorphism}

$$
\mathcal{R}_{3} / \mathcal{I}_{3} \longrightarrow A\left(\Gamma_{L}\right) \text {. }
$$

Of course this homomorphism is not an isomorphism, since the left hand side has Krull dimension 6 and the right hand side has Krull dimension 11. The picture remedies if one intersects $\Gamma_{L}$ with a certain unitary group.

The lattice $L$ admits a complex structure. We follow Kondo's description Ko2]. For this one uses the isomorphism $\mathbb{Z}^{4} \rightarrow \mathbb{Z}^{4}$ defined by the matrix

$$
\left(\begin{array}{cccc}
-1 & 0 & 2 & 0 \\
0 & 1 & 0 & 2 \\
-1 & 0 & 1 & 0 \\
0 & -1 & 0 & -1
\end{array}\right)
$$

This is an isometry

$$
\rho_{1}: U \oplus U(2) \stackrel{\sim}{\longrightarrow} U \oplus U(2) .
$$

We also use the isometry

$$
\rho_{0}: D_{4} \stackrel{\sim}{\longrightarrow} D_{4}, \quad\left(x_{1}, x_{2}, x_{3}, x_{4}\right) \longmapsto\left(x_{2},-x_{1}, x_{4},-x_{3}\right) .
$$

The direct sum $\rho:=\rho_{1} \oplus \rho_{0} \oplus \rho_{0}$ defines an isometry

$$
\rho: L \stackrel{\sim}{\longrightarrow} L .
$$


Since $\rho^{2}=-$ id we get a structure as a $\mathbb{Z}[\mathrm{i}]$-module on $L$ by defining

$$
\mathrm{i} a:=\rho(a) \text {. }
$$

One has $L \cong \mathbb{Z}[i]^{6}$. We denote by $(a, b)=q(a+b)-q(a)-q(b)$ the real bilinear form on $L$. Because of $(a, \rho(a))=0$ the pairing

$$
2\langle a, b\rangle:=(a, b)-\mathrm{i}(a, \mathrm{i} b) \quad(\Longrightarrow 2\langle a, a\rangle=(a, a))
$$

is a Hermitian form of signature $(1,5)$. Notice that we take Hermitian forms to be $\mathbb{C}$-linear in the second variable and antilinear in the first. Let $\mathrm{U}(L,\langle\cdot, \cdot\rangle)$ denote the unitary group. We set

$$
\Gamma:=\mathrm{O}^{\prime}(L) \cap \mathrm{U}(L,\langle\cdot, \cdot\rangle), \quad \Gamma[1-\mathrm{i}]:=\Gamma_{L} \cap \mathrm{U}(L,\langle\cdot, \cdot\rangle) .
$$

The natural homomorphism

$$
\Gamma / \Gamma[1-\mathrm{i}] \longrightarrow \mathrm{O}^{\prime}(L) / \Gamma_{L} \longrightarrow \mathrm{O}\left(\mathbb{F}_{2}^{6}\right) \cong S_{8}
$$

is still an isomorphism.

We denote by $\mathcal{B}$ the associated complex five-dimensional ball. It can be considered as the set of positive definite one-dimensional complex subspaces of $L \otimes_{\mathbb{Z}} \mathbb{R}$. We can intersect the Heegner divisor $\mathcal{H}_{\alpha}$ with the ball

$$
\mathcal{B}_{\alpha}:=\mathcal{H}_{\alpha} \cap \mathcal{B} \text {. }
$$

The group $\Gamma$ acts on $\mathcal{B}$ and one can consider the graded algebras of modular forms $A(\Gamma[1-\mathrm{i}])$. There is a natural restriction homomorphism $A\left(\Gamma_{L}\right) \rightarrow A(\Gamma[1-\mathrm{i}])$. Together with Lemma 2.3 we get a homomorphism $\mathcal{R}_{3} / \mathcal{I}_{3} \rightarrow A(\Gamma[1-\mathrm{i}])$.

\subsection{Theorem. The homomorphism}

$$
\mathcal{R}_{3} / \mathcal{I}_{3} \rightarrow A(\Gamma[1-i])
$$

is an isomorphism. Hence this ring of modular forms is generated by 14 modular forms with 14 defining quadratic relations. The dimension formula is given by Theorem 1.6.

Corollary. There is an induced biholomorphic map

$$
\bar{X}(8) \longrightarrow \overline{\mathcal{B} / \Gamma[1-i]},
$$

where the right hand side denotes the Baily-Borel compactification.

This result can be found in Kondo's paper Ko2. But the proof is not convincing. Kondo constructs by means of the theory of $K 3$-surfaces a period map $X(8) \longrightarrow$ $\mathcal{B} / \Gamma[1-\mathrm{i}]$. Then he wants to use an extension theorem of Borel to extend it to the compactifications. This extension theorem of Borel states essentially that a holomorphic map of a punctured disk into an arithmetic quotient $D / \Gamma$ extends to a holomorphic map of the full disk to the Satake compactification. The theorem of Borel requires that $\Gamma$ is torsion free. Otherwise it is false as, for example, $\operatorname{SL}(2, \mathbb{Z})$ shows.

The group $\Gamma[1-\mathrm{i}]$ is not torsion free. If $r$ is an element with $2\langle r, r\rangle=(r, r)=-2$, then the complex reflection

$$
x \longmapsto x+2\langle x, r\rangle x=x+(x, r) x-\mathrm{i}(x, \mathrm{i} r) x
$$

is contained in $\Gamma[1-\mathrm{i}]$. These reflections are related to the divisors $\mathcal{B}_{\alpha}=\mathcal{H}_{\alpha} \cap \mathcal{B}$. By definition this divisor consists of all $z \in \mathcal{B}$ such that

$$
(\delta, z)=(\delta, \mathrm{i} z)=0 \quad \text { for all } \quad \delta \in L^{*}, \quad(\delta, \delta)=-1, \quad \text { over } \alpha .
$$


Using the Hermitian form this is equivalent to $\langle\delta, z\rangle=0$. This we can rewrite as

$$
\langle r, z\rangle=0, \quad r:=(1-\mathrm{i}) \delta .
$$

Obviously $r \in L$ and $\langle r, r\rangle=-1$. This shows that $\mathcal{B}_{\alpha}$ is the fixed point set of a reflection inside $\Gamma[1-\mathrm{i}]$.

2.5. Remark. The zero divisor of the restriction $F_{S}=f_{S} \mid \mathcal{B}$ of the star modular form is contained in the ramification divisor of the projection $\mathcal{B} \rightarrow \mathcal{B} / \Gamma[1-\mathrm{i}]$.

Because of the ramification the star modular forms $F_{S}$, restricted to the ball, vanish at $\mathcal{B}_{\alpha}, \alpha \in S$ of order two. The ramification makes another correction in Ko2 necessary. It is possible to take a holomorphic square root $G_{S}$ of the holomorphic function $F_{S}$. But the $G_{S}$ are modular forms with nontrivial characters. Hence for two different stars $S_{1}, S_{2}$, the quotient $G_{S_{1}} / G_{S_{2}}$ is not full invariant under $\Gamma[1-\mathrm{i}]$. The stars and the tableaux are in 1-1 correspondence Ko2]. Denote by $\tau_{1}, \tau_{2}$ the tableaux which correspond to $S_{1}, S_{2}$ and by $\mu_{\tau_{1}}, \mu_{\tau_{2}}$ the corresponding Specht polynomials. Kondo claims $G_{S_{1}} / G_{S_{2}}=\mu_{\tau_{1}} / \mu_{\tau_{2}}$ (see the two lines before theorem 7.6 in [Ko2]). The correct equation is

$$
F_{S_{1}} / F_{S_{2}}=\mu_{\tau_{1}} / \mu_{\tau_{2}}
$$

The point is that the pole and zero orders of $F_{S_{1}} / F_{S_{2}}$ considered as functions on the quotient $\mathcal{B} / \Gamma[1-\mathrm{i}]$ are only one and not two.

Proof of Theorem 2.4. From the description of the zeros of the $F_{S}$ it follows that they have no common zero on the Baily-Borel compactified ball quotient. Hence one obtains a finite map

$$
\overline{\mathcal{B} / \Gamma[1-\mathrm{i}]} \longrightarrow \operatorname{proj}\left(\mathcal{R}_{3} / \mathcal{I}_{3}\right) \text {. }
$$

Since $\mathcal{R}_{3} / \mathcal{I}_{3}$ is normal, one has only to show that this map is generically injective. This follows from Kondo's comparison from $X(8)$ and $\mathcal{B} / \Gamma[1-\mathrm{i}]$ by means of $K 3$ surfaces. It can also be proven directly using the methods of [FS]. In this way one can avoid the use of $K 3$-surfaces. (It depends on the situation and also on a question of taste whether one wants to apply the theory of moduli to the theory of modular forms or conversely.)

\section{SiegEl MOdular FORMS OF GENUS THREE}

Let

$$
\mathcal{H}_{g}=\left\{Z \in \mathbb{C}^{(g, g)} ; \quad Z={ }^{t} Z, \operatorname{Im} Z>0\right\}
$$

be the Siegel half-plane of genus $g$ and

$$
\Gamma_{g}[q]=\operatorname{kernel}(\operatorname{Sp}(g, \mathbb{Z}) \longrightarrow \operatorname{Sp}(g, \mathbb{Z} / q \mathbb{Z}))
$$

be the principal congruence subgroup of level $q$ in the Siegel modular group $\Gamma_{g}:=$ $\Gamma_{g}[1]$. It acts on $\mathcal{H}_{g}$ by the usual formula $M Z=(A Z+B)(C Z+D)^{-1}$. The quotient $\mathcal{H}_{g} / \Gamma_{g}[q]$ is, by a well-known theorem of Baily, a quasi-projective variety. We denote by $\overline{\mathcal{H}_{g} / \Gamma_{g}[q]}$ its Satake compactification. This is related to the algebra of modular forms

$$
A\left(\Gamma_{g}[q]\right)=\bigoplus_{r=0}^{\infty}\left[\Gamma_{g}[q], r\right],
$$

where $\left[\Gamma_{g}[q], r\right]$ denotes the vector space of modular forms of weight $r$. These are holomorphic functions $f: \mathcal{H}_{g} \rightarrow \mathbb{C}$ with the transformation property $f(M Z)=$ 
$\operatorname{det}(C Z+D)^{r} f(Z)$ for all $M \in \Gamma_{g}[q]$ (and a regularity condition at the cusps in the case $g=1)$. Then one has

$$
\overline{\mathcal{H}_{g} / \Gamma_{g}[q]} \cong \operatorname{proj}\left(A\left(\Gamma_{g}[q]\right)\right) .
$$

We denote by $\mathcal{J}_{g} \subset \mathcal{H}_{g}$ the set of all matrices which are period matrices of hyperelliptic (nonsingular projective) curves. Then

$$
\mathcal{I}_{g}[q]=\mathcal{J}_{g} / \Gamma_{g}[q]
$$

is a quasi-projective subvariety. The variety $\mathcal{I}_{g}[1]$ is the modular variety of hyperelliptic curves. The closure

$$
\overline{\mathcal{I}_{g}[q]} \subset \overline{\mathcal{H}_{g} / \Gamma_{g}[q]}
$$

is one of its compactifications that we have to consider.

For $g \geq 3$ and $q \geq 2, \overline{\mathcal{I}_{g}[q]}$ is reducible. The number of reducible components of $\overline{\mathcal{I}_{g}[2]}$ is

$$
2^{2 g^{2}+g} \prod_{k=1}^{g}\left(1-2^{-2 k}\right) /(2 g+2) !
$$

cf. [Ts, and it is the same for all even $q$. To describe the irreducible components we have to recall the thetanullwerte, which for arbitrary $g$ are defined by

$$
\vartheta[m]=\sum_{n \in \mathbb{G}^{g}} e^{\pi \mathrm{i}\left(Z\left[n+m^{\prime} / 2\right]+\left(n+m^{\prime} / 2\right)^{\prime} m^{\prime \prime}\right)}, \quad m=\left(\begin{array}{c}
m^{\prime} \\
m^{\prime \prime}
\end{array}\right),
$$

where we use the usual notation $Z[g]=g^{\prime} Z g$. Here $m^{\prime}, m^{\prime \prime}$ are columns in $\mathbb{G}^{g}$. Up to the sign, they depend only on $m^{\prime}, m^{\prime \prime} \bmod 2$. They vanish identically if and only if ${ }^{t} m^{\prime} m^{\prime \prime}$ is odd. Hence in the case $g=3$, there are essentially 36 such thetas and 36 irreducible components of $\overline{\mathcal{I}_{3}[2]}$. By classical results a matrix $Z \in \mathcal{H}_{3}$ is in the closure of $\mathcal{J}_{3}$ if and only if the product of all 36 thetas vanishes. From this one deduces that $\mathcal{I}_{3}[2]$ has 36 irreducible components. Each of them corresponds to a so-called "even characteristic"

$$
m=\left(\begin{array}{c}
m^{\prime} \\
m^{\prime \prime}
\end{array}\right) \in \mathbb{F}_{2}^{6}, \quad{ }^{t} m^{\prime} m^{\prime \prime}=0,
$$

and is defined as

$$
\mathcal{I}_{3}[2]^{m}:=\left\{Z \in \mathcal{I}_{3}[2] ; \quad \vartheta[m](Z)=0\right\} .
$$

Here we remark that $\vartheta[m]^{4}$ is a modular form of weight 2 on $\Gamma_{2}[2]$. Hence the zero locus of $\vartheta[m]$ inside $\mathcal{I}_{3}[2]$ is well defined. We have to consider the closure

$$
\overline{\mathcal{I}}_{3}[2]{ }^{m} \subset \overline{\mathcal{H}_{3} / \Gamma_{g}[2]} .
$$

The different components are permuted under the full modular group. Hence it doesn't matter which characteristic we use. We can take for example the zero characteristic 0 . The Galois group of the covering

$$
{\overline{\mathcal{I}_{3}[2]}}^{m} \longrightarrow{\overline{\mathcal{I}_{3}[1]}}^{m}
$$

is the stabilizer of $m$ in $\operatorname{Sp}(2, \mathbb{Z} / 2 \mathbb{Z})$ with respect to the usual action of this group on characteristics:

$$
M\{m\}:={ }^{t} M^{-1} m+\left(\begin{array}{c}
\left(A^{t} B\right)_{0} \\
\left(C^{t} D\right)_{0}
\end{array}\right) \quad(\bmod 2) .
$$


Here we use the notation $A_{0}$ for the column vector built from the diagonal of a matrix $A$. In the case of zero characteristic, the stabilizer is the subgroup of $\operatorname{Sp}\left(2, \mathbb{F}_{2}\right)$, which fixes the quadratic form

$$
\mathbb{F}_{2}^{6} \longrightarrow \mathbb{F}_{2}, \quad m \longmapsto{ }^{t} m^{\prime} m^{\prime \prime} .
$$

Hence it is our orthogonal group $\mathrm{O}\left(\mathbb{F}_{2}^{6}\right)$. This is also the image of the theta group $\Gamma_{3, \vartheta}$, where

$$
\Gamma_{g, \vartheta}:=\left\{M \in \Gamma_{g} \mid \quad\left(A^{t} B\right)_{0} \equiv\left(C^{t} D\right)_{0} \equiv 0 \quad \bmod 2\right\} .
$$

Hence we have

$$
\mathrm{O}\left(\mathbb{F}_{2}^{6}\right)=\Gamma_{3, \vartheta} / \Gamma_{3}[2]
$$

Next we have to make use of the theory of Thomae, who computed the values of the theta values for hyperelliptic curves. We use (slightly modified) the beautiful approach of Mumford $[\mathrm{Mu}$. Let

$$
B=\{1, \ldots, 8\}, \quad U=\{1, \ldots, 4\} .
$$

Recall that we consider on $\mathbb{F}_{2}^{6}$ the quadratic form

$$
q: \mathbb{F}_{2}^{6} \longrightarrow \mathbb{F}_{2}, \quad q(m)={ }^{t} m^{\prime} m^{\prime \prime}
$$

and the associated pairing

$$
(m, n)=q(m+n)+q(m)+q(n)={ }^{t} m^{\prime} n^{\prime \prime}+{ }^{t} m^{\prime \prime} n^{\prime} .
$$

Their multiplicative form is

$$
e(m)=(-1)^{t} m^{\prime} m^{\prime \prime}, \quad e(m, n)=(-1)^{t} m^{\prime} n^{\prime \prime}+{ }^{t} m^{\prime \prime} n^{\prime} .
$$

Recall that a characteristic $m$ is called even if $q(m)=0(e(m)=1)$. Hence the even charactersitics are just the isotropic vectors of the quadratic space $\left(\mathbb{F}_{2}^{6}, q\right)$. There are 36 even $m$. Following Mumford $[\mathrm{Mu}$, we construct a map

$$
T \longmapsto m(T),
$$

which attaches to a subset $T \subset B$ such that $\# T$ is an even element from $\mathbb{F}_{2}^{6}$. The basic properties of this assignment are:

a) $m\left(T_{1}\right)=m\left(T_{2}\right)$ if and only if $T_{1}=T_{2}$ or $T_{1}$ is the complement of $T_{2}$. In particular, every element of $\mathbb{F}_{2}^{6}$ is in the image of the map $m$.

b) If

$$
T_{1} \triangle T_{2}=T_{1} \cup T_{2}-T_{1} \cap T_{2}
$$

denotes the symmetric difference, one has

$$
m\left(T_{1} \triangle T_{2}\right)=m\left(T_{1}\right)+m\left(T_{2}\right)+m(\emptyset) .
$$

c) The characteristic $m(T)$ is even if and only if the number of elements of $T \triangle U$ is divisible by 4 (hence it is 0,4 or 8 ).

d) $e\left(m\left(T_{1}\right)\right) e\left(m\left(T_{2}\right)\right) e\left(m\left(T_{1} \triangle T_{2}\right)\right)=(-1)^{\# T_{1} \cap T_{2}}$.

As a consequence we get

$$
\begin{aligned}
e\left(m\left(T_{1}\right), m\left(T_{2}\right)\right) & =e\left(m\left(T_{1}\right)\right) e\left(m\left(T_{2}\right)\right) e\left(m\left(T_{1}\right)+\left(m\left(T_{2}\right)\right)\right. \\
& =e\left(m\left(T_{1}\right)\right) e\left(m\left(T_{2}\right)\right) e\left(m\left(T_{1} \triangle T_{2}\right)+m(\emptyset)\right) \\
& =e\left(m\left(T_{1}\right)\right) e\left(m\left(T_{2}\right)\right) e\left(m\left(T_{1} \triangle T_{2}\right)\right) e(m(\emptyset)) e\left(m\left(T_{1} \triangle T_{2}\right), m(\emptyset)\right) \\
& =(-1)^{\# T_{1} \cap T_{2}} e(m(\emptyset)) e\left(m\left(T_{1} \triangle T_{2}\right), m(\emptyset)\right) .
\end{aligned}
$$


We observe that if we set $m(\emptyset)$ equal to the zero characteristics, then we have Mumford's procedure, but this will not be our case. To be concrete we take the following explicit realization.

$\begin{array}{cccccccc}\{1,2\} & \{1,3\} & \{1,4\} & \{1,5\} & \{1,6\} & \{1,7\} & \{1,8\} & \emptyset \\ 1 & 1 & 0 & 0 & 1 & 0 & 1 & 0 \\ 1 & 1 & 0 & 1 & 0 & 0 & 0 & 1 \\ 1 & 0 & 1 & 1 & 0 & 0 & 1 & 0 \\ 1 & 0 & 1 & 0 & 0 & 1 & 0 & 1 \\ 0 & 1 & 1 & 0 & 0 & 1 & 1 & 0 \\ 0 & 1 & 1 & 0 & 1 & 0 & 0 & 1 .\end{array}$

The first row contains subsets of $B$; the columns below are the corresponding characteristics. It is easy to verify that this assignment uniquely extends to a map with the properties a)-d). We also notice that $m(U)$ is the sum of the first three,

$$
{ }^{t} m(U)=(0,0,0,0,0,0) .
$$

The group $\operatorname{Sp}(3, \mathbb{Z} / 2 \mathbb{Z})$ acts on the set of characteristics; we recall from $[\operatorname{Ig} 1$ that $e(m)$ and $e(m, n) e(m, p) e(n, p)$ are invariant. The form $e(m, n)$ turns out to be $\mathrm{O}\left(\mathbb{F}_{2}^{6}\right)$-invariant.

The group $S_{8}$ acts on $B=\{1, \ldots, 8\}$ and hence on the pairs $\{T, B-T\}$ of subsets of even order and on $U$, so we get an induced action of $S_{8}$ on $\mathbb{F}_{2}^{6}$. We observe that $m(U)=0$ and $\#(T \triangle U)$ are invariant for the action of $S_{8}$; in fact it generates the subgroup $\mathrm{O}\left(\mathbb{F}_{2}^{6}\right)$ of $\mathrm{GL}\left(\mathbb{F}_{2}^{6}\right)$. Hence we have now constructed an explicit isomorphism

$$
S_{8} \stackrel{\sim}{\longrightarrow} \mathrm{O}\left(\mathbb{F}_{2}^{6}\right) \text {. }
$$

This isomorphism will be used in the rest of this paper. We associate to each subset $T \subset B$ of even order a monomial $D(T)$ in the ring $\mathbb{C}\left[X_{1}, \ldots, X_{8}\right]$. We have to distinguish two cases:

1) $T \triangle U \neq 4$. In this case we attach 0 .

2) $T \triangle U=4$. In this case we attach the following monomial of degree 12 :

$$
e(m(T), m(\emptyset))(-1)^{\# T \cap U} \Delta \prod_{\substack{i \in T \Delta U \\ j \notin T \Delta U}}\left(X_{i}-X_{j}\right)^{-1}, \quad \text { where } \quad \Delta=\prod_{i<j}\left(X_{i}-X_{j}\right) .
$$

Because of $D(T)=D(B-T)$ the monomial

$$
D(m):=D(m(T)) \text { for } \quad m=m(T)
$$

is well defined.

From Thomae's computation of the theta values of hyperelliptic Riemann surfaces follows (see $[\mathrm{Mu}]$ ):

3.1. Theorem (Thomae, 1870). Let $\mathbb{C}\left[\ldots \vartheta[m]^{4} \ldots\right]$ be the ring generated by the fourth powers of the 36 even thetanullwerte. Then $D$ defines a ring homomorphism

$$
D: \mathbb{C}\left[\ldots \vartheta[m]^{4} \ldots\right] \longrightarrow \mathbb{C}\left[\ldots X_{i}-X_{j} \ldots\right] .
$$

This homomorphism is equivariant with the standard action of $\mathrm{O}\left(\mathbb{F}_{2}^{6}\right)$ on the thetanullwerte and the action of $S_{8}$ on the variables $X_{i}$.

The only element of the generators which lies in the kernel is $\vartheta[0]^{4}$. But this does of course not mean that the kernel is generated by this element. 
We investigate the image. Let $m$ be the characteristic coming from the empty set, i.e. ${ }^{t} m=(0,1,0,1,0,1)$. Then we have

$$
\begin{aligned}
D\left(\vartheta[m]^{4}\right) & =\left(X_{1}-X_{2}\right)\left(X_{1}-X_{3}\right)\left(X_{1}-X_{4}\right)\left(X_{2}-X_{3}\right)\left(X_{2}-X_{4}\right)\left(X_{3}-X_{4}\right) \\
& \times\left(X_{5}-X_{6}\right)\left(X_{5}-X_{7}\right)\left(X_{5}-X_{8}\right)\left(X_{6}-X_{7}\right)\left(X_{6}-X_{8}\right)\left(X_{7}-X_{8}\right) .
\end{aligned}
$$

This is the product of the three Specht polynomials with respect to the tableaux

$$
\left(\begin{array}{llll}
1 & 3 & 5 & 7 \\
2 & 4 & 6 & 8
\end{array}\right), \quad\left(\begin{array}{llll}
1 & 2 & 5 & 6 \\
3 & 4 & 7 & 8
\end{array}\right), \quad\left(\begin{array}{llll}
1 & 2 & 5 & 6 \\
4 & 3 & 8 & 7
\end{array}\right)
$$

This homomorphism can be given explicitly as

3.2. Proposition. There is a homomorphism

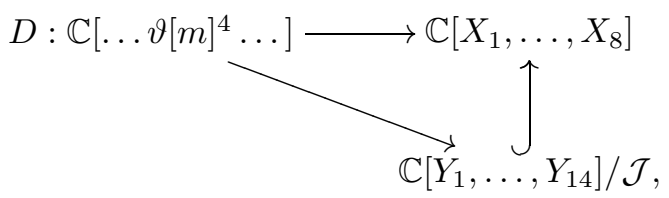

which can be characterized by the following properties:

1) Its kernel contains $\vartheta[0]^{4}$.

2) Using the notation $W_{i j}=X_{i}-X_{j}$, the image of $\vartheta[m]^{4}$ is a monomial in the $W_{i j}$. For $m^{\prime}=(0,0,0,0,0,1)$ this monomial is

$$
-W_{13} W_{16} W_{17} W_{24} W_{25} W_{28} W_{36} W_{37} W_{45} W_{48} W_{58} W_{67} .
$$

3) It has the equivariance property formulated in Theorem 3.1 .

Up to the signs, one can characterize $D$ also by the following property:

3.3. Proposition. The variable $W_{i j}$ appears in the monomial $D\left(\vartheta[m]^{4}\right), m \neq 0$, if and only if $m$ is orthogonal to the (odd) characteristic $m\left(T_{i j}\right)$, where $T_{i j}$ is defined by

$$
T_{i j} \triangle U=\{i, j\} .
$$

Proof. It is easy to verify this for the given $m$. The general case follows then because $\mathrm{O}\left(\mathbb{F}_{2}^{6}\right)$ acts transitively on the set of nonzero even characteristics and since the description is $\mathrm{O}\left(\mathbb{F}_{2}^{6}\right)$-equivaraint.

Finally we mention an observation of Igusa Ig2:

3.4. Proposition. D extends to a ring homomorphism

$$
D: A\left(\Gamma_{3}[2]\right) \longrightarrow \mathbb{C}\left[Y_{1}, \ldots, Y_{14}\right] / \mathcal{J} .
$$

An element $f$ belongs to the kernel if and only if it vanishes on $\mathcal{I}_{3}[2]^{0}$.

Recall that on the right hand side we have the ring of Specht polynomials from section 1 , which also can be identified with the ring of ball modular forms $\mathcal{R}_{3} / \mathcal{I}_{3}$ from section 2 . 


\section{A structure theorem}

Runge Ru2 has described generators for the ring of Siegel modular forms of genus three and level two with respect to the trivial character. The restriction to the trivial character implies that he obtains modular forms of even weight. For our purposes it is useful to include modular forms of odd weight. This means a slight extension of Runge's results.

Runge gave explicitly 15 generators in weight 2 and 15 generators in weight 4 . He expressed them as explicit polynomials in the 8 theta constants of the second kind:

$$
f_{a}(Z)=\sum_{g \in \mathbb{Z}^{3}} e^{2 \pi \mathrm{i} Z[g+a / 2]}, \quad a \in(\mathbb{Z} / 2 \mathbb{Z})^{3} .
$$

We recall the relation to the $\vartheta[m]$ :

$$
\vartheta^{2}\left[\begin{array}{c}
m^{\prime} \\
m^{\prime \prime}
\end{array}\right]=\sum_{a \in \mathbb{F}_{2}^{3}}(-1)^{t} m^{\prime} m^{\prime \prime} f_{m^{\prime}+a} f_{a}, \quad f_{a} f_{b}=\frac{1}{8} \sum_{c \in \mathbb{F}_{2}^{3}}(-1)^{t} a c \vartheta^{2}\left[\begin{array}{c}
a+b \\
c
\end{array}\right] .
$$

The classical Schottky relation $R$ can be written as a polynomial in degree 16 of the $f_{a}$. Runge proved the basic result that this is a defining relation in the ring $\mathbb{C}\left[f_{a}\right]$ and moreover that this ring is normal. This implies that the subring $\mathbb{C}\left[f_{a} f_{b}\right]$ of forms of integral weight is the full ring of modular forms of integral weight and trivial character with respect to Runge's group $\Gamma_{3}^{*}[2,4]$. This is the unique subgroup of Igusa's group $\Gamma_{3}[2,4]$ which does not contain the negative unit matrix. Recall that the Igusa group $\Gamma_{g}[q, 2 q]$ is the subgroup of all $M \in \Gamma_{g}[q]$ such that $A^{t} B / q$ and $C^{t} D / q$ have even diagonal. Hence the theta group is $\Gamma_{g, \vartheta}=\Gamma_{g}[1,2]$.

We recall briefly the way Runge describes the action of the modular group on the $f_{a}$. He introduces the following finite group $H_{3}$ of $8 \times 8$ matrices (indexed by the 8 elements from $\mathbb{F}_{2}^{3}$ ). The first generator is

$$
\tilde{S}=\left(\frac{1+\mathrm{i}}{2}\right)^{3}\left((-1)^{t} a b\right) .
$$

To every integral diagonal $3 \times 3$ matrix one attaches the diagonal matrix $\tilde{T}_{S}$ with diagonal entries $\mathrm{i}^{S[a]}$. By definition the group $H_{3}$ is generated by the matrices $\tilde{I}$ and by the $\tilde{T}_{S}$. The negative unit matrix is contained in $H_{3}$. If we map $I$ to $\tilde{I}$ and $T_{S}$ to $\tilde{T}_{S}$ we obtain a homomorphism

$$
\Gamma_{3} \longrightarrow H_{3} / \pm
$$

The group $H_{3}$ and this homomorphism reflect the action of modular substitutions on the 8-dimensional space $\sum \mathbb{C} f_{a}$. The action of a modular substitution $M$ on this space is given by its image in $H_{3} / \pm$. Of course one has to take care about a sign ambiguity, which is typical for the theta transformation formalism. The kernel of this homomorphism $\Gamma_{3} \longrightarrow H_{3} / \pm$ is Runge's congruence subgroup $\Gamma^{*}[2,4]$.

There is also a surjective homomorphism

$$
H_{3} \longrightarrow \operatorname{Sp}\left(3, \mathbb{F}_{2}\right),
$$

which sends $\tilde{I}$ and $\tilde{T}_{S}$ to the cosets of $I$ and $T_{S}$. The kernel of the homomorphism is

$$
N_{3}:=\left\langle\mathrm{i} E, \tilde{T}_{2 S}, \tilde{I}^{-1} \tilde{T}_{2 S} I, \quad(S \text { integral })\right\rangle,
$$


as correctly stated in Runge's first paper [Ru1]. In the second part of his paper Ru2 Runge claims that $N_{3}$ agrees with

$$
N_{3}^{\prime}:=\left\langle\tilde{T}_{2 S}, \tilde{I}^{-1} \tilde{T}_{2 S} I, \quad(S \text { integral })\right\rangle .
$$

But this is not the case, since $N_{3}^{\prime} \subset N_{3}$ is a subgroup of index two which doesn't contain i $E$. It contains only $-E$.

This small correction allows us to modify Runge's results on modular forms of level two by introducing the theta character $v_{\vartheta}$. This is the character of the modular form $\vartheta[0]^{2}$, which lives on the theta group $\Gamma_{3, \vartheta}=\Gamma_{3}[1,2]$. This group contains the principal congruence group of level two $\Gamma_{3}[2]$. We consider the ring

$$
A\left(\Gamma_{3}[2]\right):=\bigoplus_{r \in \mathbb{G}}\left[\Gamma_{3}[2], r, v_{\vartheta}^{r}\right] .
$$

This ring also contains nonzero forms of odd weight. Now a slight modification of Runge's results gives:

4.1. Proposition. Let $F_{a}$ be a formal variable for $a \in \mathbb{F}_{2}^{3}$. We consider the polynomial ring $\mathbb{C}\left[F_{a}\right]$. The group $H_{3}$ acts on this ring through the standard linear action on the space $\mathbb{C} F_{a}$. There exists a polynomial $R$ of degree 16 in the $F_{a}$, which describes the Schottky relation. Since $R$ is invariant under $H_{3}$ the group $N_{3}^{\prime}$ acts also on $\mathbb{C}\left[F_{a}\right] /(R)$. There is a natural isomorphism

$$
\left(\mathbb{C}\left[F_{a}\right] /(R)\right)^{N_{3}^{\prime}}=\left(\mathbb{C}\left[F_{a}\right]^{N_{3}^{\prime}}\right) /(R) \stackrel{\sim}{\longrightarrow} A\left(\Gamma_{3}[2]\right),
$$

which replaces $F_{a}$ by $f_{a}$.

From this result one can derive:

4.2. Proposition. The ring $A\left(\Gamma_{3}[2]\right)$ is generated by

1 form of weight 1 (namely $\vartheta[0]^{2}$ ), which belongs to the theta group,

14 forms of weight 2 ,

14 forms of weight 3 ,

1 form of weight 4 , which belongs to the theta group.

The Hilbert function of the ring is $\left(1-z^{8}\right)$ times

$$
\frac{1-3 z+13 z^{2}-17 z^{3}+44 z^{4}-17 z^{5}+13 z^{6}-3 z^{7}+z^{8}}{\left(1-z^{2}\right)^{4}(1-z)^{4}},
$$

which is

$$
\begin{aligned}
& 1+z+15 z^{2}+29 z^{3}+135 z^{4}+310 z^{5}+870 z^{6}+1830 z^{7} \\
& +3992 z^{8}+7534 z^{9}+14142 z^{10}+\cdots .
\end{aligned}
$$

If one takes the subring of $A\left(\Gamma_{3}[2]\right)$, which is generated by forms of even weight, one obtains Runge's theorem 5.3 in [Ru2].

\section{The RING OF HYPERELliptiC MODUlar FORMS OF GENUS 3}

The factor group $\Gamma_{3} / \Gamma_{3}[2]$ is isomorphic to $\operatorname{Sp}(3, \mathbb{Z} / 2 \mathbb{Z})$. This group contains naturally the orthogonal group $\mathrm{O}\left(\mathbb{F}_{2}^{6}\right)$ (quadratic form $m_{1} m_{4}+m_{2} m_{5}+m_{3} m_{6}$ ). Recall that the inverse image of $\mathrm{O}\left(\mathbb{F}_{2}^{6}\right)$ in the Siegel modular group is the theta group $\Gamma_{3, \vartheta}$. The group $\mathrm{O}\left(\mathbb{F}_{2}^{6}\right)$ has a simple subgroup of index two, which we denote by $\mathrm{SO}\left(\mathbb{F}_{2}^{6}\right)$. Its inverse image is a subgroup of index two $\Gamma_{3, \vartheta}^{\prime} \subset \Gamma_{3, \vartheta}$. 
It is important to find a natural system of generators of the space $\left[\Gamma_{3}[2], 2\right]$. Actually we will construct 15 forms $\Theta_{1}, \ldots, \Theta_{15}$, which generate this space and which have the property that they are permuted under the action of the group $\Gamma_{3, \vartheta}^{\prime}$. For their construction we use the 36 theta constants of the first kind $\vartheta[m]$.

We have thirty maximal totally isotropic spaces contained in $\mathbb{F}_{2}^{6}$. For such a subspace $A$ we set

$$
\Theta=\sum_{i=1}^{7} \vartheta\left[m_{i}\right]^{4},
$$

where $m_{1}, \ldots, m_{7}$ are the nonzero elements of $A$. Of course the orthogonal group acts transitively on the set of totally isotropic spaces. But this is not the case for the subgroup $\mathrm{SO}\left(\mathbb{F}_{2}^{6}\right)$. We use the well-known fact that under this group the totally isotropic spaces split into two orbits. (Two spaces are in the same orbit if and only if their intersection has even dimension.) We give the list of the subspaces in one of the two orbits. We give their entries different from 0 ordered lexicographically; i.e., $m$ is replaced by the digit $2^{5} m_{1}+2^{4} m_{2}+2^{3} m_{3}+2^{2} m_{4}+2 m_{5}+m_{6}$. The fifteen subspaces are:

$$
\begin{array}{ll}
A_{1}=[6,8,14,48,54,56,62] & A_{2}=[6,10,12,49,55,59,61] \\
A_{3}=[7,24,31,40,47,48,55] & A_{4}=[7,27,28,42,45,49,54] \\
A_{5}=[4,8,12,16,20,24,28] & A_{6}=[4,10,14,17,21,27,31] \\
A_{7}=[5,16,21,40,45,56,61] & A_{8}=[5,17,20,42,47,59,62] \\
A_{9}=[2,8,10,32,34,40,42] & A_{10}=[2,12,14,33,35,45,47] \\
A_{11}=[3,24,27,32,35,56,59] & A_{12}=[3,28,31,33,34,61,62] \\
A_{13}=[1,16,17,32,33,48,49] & A_{14}=[1,20,21,34,35,54,55] \\
A_{15}=[1,2,3,4,5,6,7] . &
\end{array}
$$

For each $A_{i}$ we denote by $\Theta_{i}$ the corresponding sum of fourth powers of the thetanullwerte.

5.1. Proposition. The forms $\Theta_{1}, \ldots, \Theta_{15}$ give a basis of the space $\left[\Gamma_{3}[2], 2\right]$. As a consequence of the quartic Riemann relations, the form

$$
\Theta_{1}+\cdots+\Theta_{15}
$$

vanishes on the hyperelliptic component defined by $\vartheta[0]=0$.

We are interested in the restriction of the ring $A\left(\Gamma_{3}[2]\right)$ to one of the hyperelliptic components. We take the component defined by the zero locus of $\vartheta[0]$. It turns out that not only $\vartheta[0]^{2}$ but also the other $\Gamma_{3, \vartheta}$ invariant form in Proposition 4.2 of weight 4 vanishes on this component. It is possible to write them explicitly as polynomials in the $F_{a}$. The expression for $\vartheta[0]^{2}$ is very simple, namely,

$$
P:=F_{0}^{2}+F_{1}^{2}+F_{2}^{2}+F_{3}^{2}+F_{4}^{2}+F_{5}^{2}+F_{6}^{2}+F_{7}^{2},
$$

where the $F_{a}$ have been ordered lexicographically; i.e., $a$ has been replaced by the digit $4 a_{1}+2 a_{2}+a_{3}$. The expression $Q$ as a polynomial in the $F_{a}$ of the second one is more complicated. There is no need to print it here. Of course $Q$ is determined only $\bmod P^{4}$, but the ideal $(P, Q)$ is well defined.

\subsection{Proposition. The ring}

$$
\mathbb{C}\left[F_{a}\right] /(P, Q)
$$

is normal of Krull dimension 6. 
Proof. Since it is a complete intersection, one has only to show that the codimension of the singular locus is at least two. This can be checked with a computer.

We need the invariant ring

$$
B\left(\Gamma_{3}[2]\right):=\left(\mathbb{C}\left[F_{a}\right] /(P, Q)\right)^{N_{3}^{\prime}}=\mathbb{C}\left[F_{a}\right]^{N_{3}^{\prime}} /(P, Q) .
$$

The zero locus of $\vartheta[0]$ is an irreducible subset of $\mathcal{H}_{3} / \Gamma_{3}[2]$. The restriction of the ring of modular forms $A\left(\Gamma_{3}[2]\right)$ to this hyperelliptic component hence is

$$
A\left(\Gamma_{3}[2]\right) /\left(\operatorname{rad}\left(\left(\vartheta[0]^{2}\right)\right)\right) .
$$

5.3. Theorem. The restriction of the ring of modular forms $A\left(\Gamma_{3}[2]\right)$ to the hyperelliptic component, set theoretically defined by $\vartheta[0]=0$, is

$$
A\left(\Gamma_{3}[2]\right) /\left(\operatorname{rad}\left(\left(\vartheta[0]^{2}\right)\right)\right) \cong B\left(\Gamma_{3}[2]\right)=\mathbb{C}\left[F_{a}\right]^{N_{3}^{\prime}} /(P, Q) .
$$

It is generated by 14 forms of weight 2 and 14 forms of weight 3 . The Hilbert function of this ring is the product of the Hilbert function of $A\left(\Gamma_{3}[2]\right)$ (see Proposition 4.2) with $(1-z)\left(1-z^{4}\right) /\left(1-z^{8}\right)$,

$$
1+14 z^{2}+14 z^{3}+105 z^{4}+175 z^{5}+546 z^{6}+946 z^{7}+2057 z^{8}+\cdots .
$$

For the proof we have to describe the relations between the generators. For this we need a method which allows us to decide whether a system $f_{1}, \ldots, f_{m}$ of homogeneous polynomials in the variables $F_{a}$ is linearly independent in the quotient $\mathbb{C}\left[F_{a}\right] /(P, Q)$. Of course we assume that all $f_{i}$ have the same degree. We used the following method: First of all, we computed a Groebner basis of the ideal $(P, Q)$, using the computer algebra SINGULAR. Then we computed the normal forms $g_{1}, \ldots, g_{m}$ of the $f_{i}$ with respect to this basis. The point now is that the $f_{1}, \ldots, f_{m}$ are linearly independent in the factor ring $\mathbb{C}\left[F_{a}\right] /(P, Q)$ if and only if the $g_{1}, \ldots, g_{m}$ are linearly independent in the polynomial ring $\mathbb{C}\left[F_{a}\right]$. This is of course a problem in linear algebra. Using this method we proved:

5.4. Proposition. The space of forms in $B\left(\Gamma_{3}[2]\right)$ of weight four, which is generated by products of two forms of weight two has dimension 105. The space of forms of weight six, which is generated by products of three forms of weight two has dimension 546 .

1. Corollary. The 14 forms of weight two generate in $B\left(\Gamma_{3}[2]\right)$ all forms of weights 4 and 6 .

2. Corollary. There are 560 monomials of degree 3 in the 14 generators of weight two. Hence there is a 14-dimensional space of cubic relations.

It seems to be natural now to consider the subring

$$
B^{\prime}\left(\Gamma_{3}[2]\right) \subset B\left(\Gamma_{3}[2]\right)
$$

generated by the 14 elements of weight 2. Because of Proposition 5.4 one can conjecture that both rings agree in even weights. We will see that this is true (Theorem 5.8).

Several times a 14-dimensional space occurred. This depends on the fact that the group $\mathrm{O}\left(6, \mathbb{F}_{2}\right) \cong S_{8}$ admits an irreducible 14-dimensional representation. There are actually two isomorphism classes which are intertwined by the signum character. All 14-dimensional spaces, which occurred so far, are irreducible. Next we describe the 14 cubic relations. 
5.5. Lemma. There exists a ternary polynomial in the $\Theta_{i}$, such that its orbit under the group $\Gamma_{3, \theta^{\prime}}$ gives a system of 14 relations, which generate the whole space of relations in this weight Proposition 5.4.

Such a polynomial in the $\Theta_{i}$ can be written explicitly, but the expression is too complicated to print it here. We just mention that one can find it by restricting a relation, which follows from the quartic Riemann relations, to the hyperelliptic component.

The relations of degree $\leq 3$ above do not generate the ideal of all relations. We also need some quartic relations. To compute them one needs the expressions of the $\vartheta[m]^{4}$ as linear combinations of the $\Theta_{1}, \ldots, \Theta_{15}$. Here is one:

$$
6 \vartheta\left[\begin{array}{ll}
0 & 0 \\
0 & 0 \\
0 & 1
\end{array}\right]^{4}=\Theta_{13}+\Theta_{14}+\Theta_{15}
$$

(We use the notation $\vartheta[m]=\vartheta\left[m^{\prime}, m^{\prime \prime}\right]$.) The others are obtained by applying $\mathrm{O}\left(\mathbb{F}_{2}^{6}\right)$. Actually there will be two types of quartic relations between the $\vartheta[\mathrm{m}]^{4}$. Let $M$ be a set of characteristics. We denote by $\vartheta[M]$ the product of all $\vartheta[m]$ with $m \in M$. Let $M$ now be a two-dimensional $\mathbb{F}_{2}$-vector space of characteristics. There are three cosets $M, a+M+a, b+M$ containing only even characteristics. We have the Riemann relation

$$
\vartheta[M]=\vartheta[M+a] \pm \vartheta[M+b] .
$$

If we set $\vartheta[0]$ to zero we obtain the relation $\vartheta[a+M]^{4}=\vartheta[b+M]^{4}$.

5.6. Proposition. There are 210 relations between the $\Theta_{i}$ (considered on the hyperelliptic component $\vartheta[0](\tau)=0)$ which come from the relations

$$
\vartheta[a+M]^{4}=\vartheta[b+M]^{4} .
$$

Here $M$ is a two-dimensional $\mathbb{F}_{2}$-vector space of characteristics and $a+M$ and $b+M$ are the two orbits consisting only of even characteristics.

One can expand these relations as quartic polynomials in the $\Theta_{i}$. We don't print them here.

We also need quartic relations which contain only squares of thetas. They are of the form

$$
\vartheta\left[m_{1}\right]^{2} \vartheta\left[m_{2}\right]^{2} \pm \vartheta\left[m_{3}\right]^{2} \vartheta\left[m_{4}\right]^{2}=\vartheta\left[m_{5}\right]^{2} \vartheta\left[m_{6}\right]^{2} \pm \vartheta\left[m_{7}\right]^{2} \vartheta\left[m_{8}\right]^{2} .
$$

Now assuming $\vartheta\left[m_{1}\right]=\vartheta[0]=0$ we have

$$
\pm \vartheta\left[m_{3}\right]^{2} \vartheta\left[m_{4}\right]^{2}=\vartheta\left[m_{5}\right]^{2} \vartheta\left[m_{6}\right]^{2} \pm \vartheta\left[m_{7}\right]^{2} \vartheta\left[m_{8}\right]^{2} .
$$

Squaring we get

$$
\vartheta\left[m_{3}\right]^{4} \vartheta\left[m_{4}\right]^{4}-\vartheta\left[m_{5}\right]^{4} \vartheta\left[m_{6}\right]^{4}-\vartheta\left[m_{7}\right]^{4} \vartheta\left[m_{8}\right]^{4}= \pm 2 \vartheta\left[m_{5}\right]^{2} \vartheta\left[m_{6}\right]^{2} \vartheta\left[m_{7}\right]^{2} \vartheta\left[m_{8}\right]^{2} .
$$

Squaring again we get a quartic relation among $\vartheta[m]^{4}$. This can be expressed in the $\Theta_{i}$. The total number of these relations between the $\Theta_{i}$ is 105 .

5.7. Proposition. The relations of the type (considered in $B\left(\Gamma_{3}[2]\right)$ )

$$
\left(\vartheta\left[m_{3}\right]^{4} \vartheta\left[m_{4}\right]^{4}-\vartheta\left[m_{5}\right]^{4} \vartheta\left[m_{6}\right]^{4}-\vartheta\left[m_{7}\right]^{4} \vartheta\left[m_{8}\right]^{4}\right)^{2}=4 \vartheta\left[m_{5}\right]^{4} \vartheta\left[m_{6}\right]^{4} \vartheta\left[m_{7}\right]^{4} \vartheta\left[m_{8}\right]^{4}
$$

give 105 relations between the $\Theta_{i}$. 
We will not give the explicit polynomials in the $\Theta_{i}$. The relations described so far are still not all. The Schottky relation

$$
\left(\sum_{m} \vartheta[m]^{8}\right)^{2}-8 \sum_{m} \vartheta[m]^{16}
$$

is an extra relation. The expansion of this relation as a polynomial in the $\Theta_{i}$ is very big. We don't print it.

Remember that we now have the following system of relations between the 15 functions $\Theta_{i}$ considered in $B\left(\Gamma_{3}[2]\right)$ :

1) one linear relation,

2) 14 cubic relations,

3) two systems of quartic relations, one consisting of 105, the other of 210 relations,

4) an extra quartic relation.

This set of relations is permuted under the action of $\Gamma_{3, \vartheta}^{\prime}$. We consider the ideal $\mathcal{R}$ generated by all these relations.

Using the computer algebra system SINGULAR one can get a Gröbner basis of this ideal, which allows us to do several computations; for example, it is possible to get the Hilbert function of the algebra

$$
\mathbb{C}\left[T_{1}, \ldots, T_{15}\right] / \mathcal{R} .
$$

Here the $T_{i}$ are formal variables which stand for the $\Theta_{i}$.

SINGULAR gives the following Hilbert function:

$$
\left(1+8 z^{2}+36 z^{4}+106 z^{6}+91 z^{8}+14 z^{10}\right) /\left(1-z^{2}\right)^{6} .
$$

(The weight of $\Theta_{i}$ is two.) One checks immediately that this series agrees with the even part of the Hilbert series of the ring $B\left(\Gamma_{3}[2]\right)$ as has been described in Proposition 6.2. Hence we obtain that our ideal describes all relations.

We mention that our system of relations is not minimal. The system of relations has the advantage of being invariant under the group $\Gamma_{3, \vartheta}^{\prime}$.

The final result is:

5.8. Theorem. The subring $B^{(2)}\left(\Gamma_{3}[2]\right)$ of forms of even weight of the ring

$$
B\left(\Gamma_{3}[2]\right)=A\left(\Gamma_{3}[2]\right) /\left(\operatorname{rad}\left(\left(\vartheta[0]^{2}\right)\right)\right)
$$

is generated by 15 forms $\Theta_{1}, \ldots, \Theta_{15}$. The ideal of relations is generated by one linear relation, 14 ternary relations and 127 quartic relations: The Hilbert function is given by the formula

$$
\begin{aligned}
& \frac{1+8 z^{2}+36 z^{4}+106 z^{6}+91 z^{8}+14 z^{10}}{\left(1-z^{2}\right)^{6}} \\
& =1+14 z^{2}+105 z^{4}+546 z^{6}+2057 z^{8}+6062 z^{10}+14945 z^{12} \\
& +32306 z^{14}+63217 z^{16}+114478 z^{18}+\cdots
\end{aligned}
$$

The projective variety of this ring is the hyperelliptic component in the Satake compactified Siegel modular variety $\overline{\mathcal{H}_{3} / \Gamma_{3}[2]}$. Hence this closure is a normal variety. 


\section{Blowing UP}

We want to investigate the homomorphism

$$
B^{(2)}\left(\Gamma_{3}[2]\right)=\mathbb{C}\left[\ldots \vartheta[m]^{4} \ldots\right] \longrightarrow \mathbb{C}\left[Y_{1}, \ldots, Y_{14}\right] / \mathcal{J} .
$$

Here the $\vartheta^{4}[\mathrm{~m}]$ are understood as forms on the hyperelliptic component. Recall that the $\vartheta^{4}[m]$ can be expressed by means of the $\Theta_{i}$. Hence the ideal of relations between the $\vartheta^{4}[\mathrm{~m}]$ (restricted to the hyperelliptic component) is known from Theorem [5.8. The right hand side is the graded algebra of the ball quotient. The ideal $\mathcal{J}$ of relations has also been described explicitly in Lemma 1.1 Recall that we have an explicit isomorphism $\mathrm{O}\left(\mathbb{F}_{2}^{6}\right) \cong S_{8}$. The group $\mathrm{O}\left(\mathbb{F}_{2}^{6}\right)$ acts transitively on the even characteristics $m \neq 0$. The group $S_{8}$ acts linearly on the $Y_{i}$. (They are polynomials in 8 variables $X_{1}, \ldots, X_{8}$ on which the group $S_{8}$ acts by permutation.)

Using Thomae's theorem 3.1 and the expressions of the $Y_{i}$ as polynomials in the $X_{i}$ (section 1) one can compute explicit expressions of the images of the $\vartheta[m]^{4}$. The base locus ideal is the ideal generated by the images. Since the above homomorphism is $\mathrm{O}\left(\mathbb{F}_{2}^{6}\right)=S_{8}$-equivariant, it is sufficient to give the image of one $\vartheta[m]^{4}$.

6.1. Lemma. The homomorphism

$$
\mathbb{C}\left[\ldots \vartheta[m]^{4} \ldots\right] \longrightarrow \mathbb{C}\left[Y_{1}, \ldots, Y_{14}\right] / \mathcal{J}
$$

is defined by

$\vartheta\left[\begin{array}{ll}0 & 0 \\ 0 & 0 \\ 0 & 1\end{array}\right]^{4} \longmapsto\left(Y_{1}-Y_{10}+Y_{11}-Y_{14}\right)\left(Y_{1}-Y_{2}-Y_{6}+Y_{7}+Y_{8}-Y_{9}+Y_{11}-Y_{13}\right)\left(Y_{8}-Y_{9}\right)$. The others are obtained by applying $S_{8}$.

The base locus is defined by the ideal which is generated by the images of the $\vartheta[m]^{4}$.

6.2. Proposition. The ideal, which is generated by the images of the $\vartheta[m]^{4}$ in the ring $\mathbb{C}\left[Y_{1}, \ldots, Y_{14}\right] / \mathcal{J}$ is the intersection of 56 ideals which are generated by linear forms. One of them is

$$
\left(Y_{2}, Y_{3}, Y_{5}, Y_{6}, Y_{8}, Y_{9}, Y_{10}, Y_{11}, Y_{13}, Y_{14}\right) .
$$

The others are obtained by applying $S_{8}$.

This can be verified by a SINGULAR calculation. However, in the last section, cf. Proposition 7.13, we shall give a more geometric and intrinsic description of the components of the zero set of the ideal generated by the images of the $\vartheta[m]^{4}$.

Let $\mathfrak{A}$ be an ideal in a (commutative and with unity) Noetherian ring $A$. The blowup of $A$ along $\mathfrak{A}$ is the graded $A$-algebra

$$
\operatorname{Bl}(A, \mathfrak{A}):=\bigoplus_{n=0}^{\infty} \mathfrak{A}^{n} .
$$

We can consider $\operatorname{Bl}(A, \mathfrak{A})$ as a subring of $A[T]$, using the embedding

$$
\mathrm{Bl}(A, \mathfrak{A}) \longrightarrow A[T], \quad a \in \mathfrak{A}^{n} \longmapsto a T^{n} .
$$

Now we assume

$$
A=\mathbb{C}\left[X_{1}, \ldots, X_{n}\right] / \mathfrak{a} .
$$


We choose polynomials $Q_{1}, \ldots, Q_{m}$ in $\mathbb{C}\left[X_{1}, \ldots, X_{n}\right]$, whose images in $A$ generate $\mathfrak{A}$. We consider the homomorphism of polynomial rings

$$
\mathbb{C}\left[X_{1}, \ldots, X_{n}, Y_{1}, \ldots, Y_{m}\right] \longrightarrow \mathbb{C}\left[X_{1}, \ldots, X_{n}, T\right], \quad Y_{i} \longmapsto Q_{i} T
$$

We denote by $\tilde{\mathfrak{A}}$ the inverse image of the ideal generated by $\mathfrak{a}$. Then we have

$$
\operatorname{Bl}(A, \mathfrak{A})=\mathbb{C}\left[X_{1}, \ldots, X_{n}, Y_{1}, \ldots, Y_{m}\right] / \tilde{\mathfrak{A}} .
$$

We need a modification of this construction. Assume that $A$ already is a graded algebra and $\mathfrak{A}$ a graded ideal. Then $\operatorname{Bl}(A, \mathfrak{A})$ is a bigraded algebra, and one can define in an obvious way the projective variety $\operatorname{biproj}(\operatorname{Bl}(A, \mathfrak{A}))$ together with a morphism

$$
\operatorname{biproj}(\operatorname{Bl}(A, \mathfrak{A})) \longrightarrow \operatorname{proj} A \text {. }
$$

We describe this map in coordinates. For simplicity we assume that the degree of the $X_{i}$ are one and that the $Q_{i}$ all are of the same degree. The variety $\operatorname{biproj}(\operatorname{Bl}(A, \mathfrak{A}))$ then consists of all pairs $([x],[y]),[x] \in P^{n-1}(\mathbb{C}),[y] \in P^{m-1}(\mathbb{C})$ such that $P(x, y)=0$ for all polynomials $P(X, Y) \in \tilde{\mathfrak{A}}$ which are homogeneous in $X=\left(X_{1}, \ldots, X_{n}\right)$ and $Y=\left(Y_{1}, \ldots, Y_{m}\right)$.

We want to apply this in the following situation. We consider the homomorphism

$$
A=\mathbb{C}\left[\ldots \vartheta[m]^{4} \ldots\right] \longrightarrow B=\mathbb{C}\left[Y_{1}, \ldots, Y_{14}\right] / \mathcal{J}
$$

Here the $\vartheta[m]^{4}$ are understood to be restricted to the hyperelliptic component; hence $\vartheta[0]^{4}=0$. The ideal which we want to blow up is generated by the images of the $\vartheta[m]^{4}$ in $B$. We have shown that this ideal is generated by 56 linear forms. We denote the blowup of this ideal by $B^{*}$ and the associated variety by $Y^{*}$. This is a model which lies over the Siegel model $X=\operatorname{proj}(A)$ and over the ball model $Y=\operatorname{proj}(B)$ :

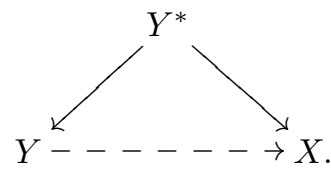

We want to get explicit information about the model $Y^{*}$. Actually it is too difficult to blow up the 56 components of the base locus in one step. But this is not necessary. To get information about the blowup of a small neighborhood of a given point in $Y$, it is sufficient to blow up those of the linear components which contain this point. To make use of this we need the intersection behavior of the 56. A direct computation shows:

6.3. Lemma. When a subset of the 56 linear components has a common intersection, it intersects also in a cusp. In each cusp meet 8 of the linear components. 
For example, the 8 components defined by the following ideals (each generated by 10 linear forms)

$$
\begin{aligned}
& \left(Y_{2}, Y_{3}, Y_{5}, Y_{6}, Y_{8}, Y_{9}, Y_{10}, Y_{11}, Y_{13}, Y_{14}\right) \\
& \left(Y_{1}, Y_{2}, Y_{3}-Y_{11}+Y_{14}, Y_{4}-Y_{12}+Y_{14}, Y_{5}, Y_{6}-Y_{11}, Y_{7}-Y_{12},\right. \\
& \left.\quad Y_{8}-Y_{9}, Y_{10},-Y_{14}+Y_{13}\right) \\
& \left(Y_{1}, Y_{2}, Y_{3}, Y_{5}-Y_{13}+Y_{14}, Y_{6}-Y_{13}, Y_{7}-Y_{12}, Y_{8}-Y_{13},\right. \\
& \left.\quad Y_{9}-Y_{14}, Y_{10}-Y_{13}+Y_{14}, Y_{11}-Y_{13}\right) \\
& \left(Y_{1}, Y_{2}-Y_{11}+Y_{13}, Y_{3}-Y_{11}+Y_{13}, Y_{4}-Y_{12}, Y_{5}-Y_{11}, Y_{6}-Y_{11},\right. \\
& \left.\quad Y_{7}-Y_{12}, Y_{9}, Y_{10}-Y_{11}, Y_{14}\right) \\
& \left(Y_{1}, Y_{2}, Y_{3}, Y_{4}-Y_{7}, Y_{5}, Y_{6}, Y_{10}, Y_{11}, Y_{13}, Y_{14}\right), \\
& \left(Y_{1}-Y_{10}-Y_{14}, Y_{2}-Y_{10}-Y_{14}, Y_{3}, Y_{4}-Y_{9}-Y_{12}+Y_{14}, Y_{5}-Y_{10},\right. \\
& \left.Y_{6}, Y_{7}-Y_{9}-Y_{12}+Y_{14}, Y_{8}, Y_{11}, Y_{13}\right), \\
& \left(Y_{2}-Y_{10}, Y_{3}-Y_{11}, Y_{4}-Y_{12}, Y_{5}-Y_{10}, Y_{6}-Y_{11}, Y_{7}-Y_{12},\right. \\
& \left.Y_{8}, Y_{9}, Y_{13}, Y_{14}\right) \\
& \left(Y_{1}+Y_{7}-Y_{12}, Y_{2}+Y_{13}-Y_{14}, Y_{3}+Y_{13}, Y_{4}-Y_{12}+Y_{14},\right. \\
& \left.Y_{5}, Y_{6}, Y_{8}, Y_{9}, Y_{10}, Y_{11}\right)
\end{aligned}
$$

meet in a cusp with the coordinates $[0,0,0,1,0,0,1,0,0,0,0,1,0,0]$.

The intersection of the 8 ideals that are generated by these linear forms is generated by 17 elements of degree $\leq 2$. We will not print them and mention just that this ideal is simple enough to be blown up by means of SINGULAR. In this way we obtain for each cusp $s$ a partial blowup $Y_{s}^{*}$. They exhaust $Y^{*}$ in the following sense. Consider for each cusp the complement of the remaining $48=56-8$ components in $Y_{s}^{*}$. These are quasi-projective varieties which give an open covering of $Y^{*}$. This means that we have a description of $Y^{*}$ by explicit equations. We will not print them here, but give some consequences.

6.4. Proposition. The model $Y^{*}$ is smooth outside the inverse images of the cusps. It is not smooth everywhere. The inverse image of a cusp is a two-dimensional irreducible variety.

To desingularize one needs one further blowup:

6.5. Proposition. The blowup of $Y^{*}$ along the inverse images of the cusps is a nonsingular model $\tilde{Y}$.

For the proof it is convenient to proceed slightly differently. We first blow up the cusps and then the inverse images of the 56 linear spaces. The result is the same. Direct computation shows:

6.6. Proposition. The blowup of the ball model $Y$ along the cusps is a smooth model $Y^{\prime}$. The inverse images of the cusps are irreducible. The intersection of the strict transforms of any 3 of the 56 linear subspaces of $Y$ is empty.

Since $Y^{\prime}$ is smooth, the inverse image of the union of the 56 linear spaces and the union of the strict transforms only differ by an invertible ideal. Since the blowup of two ideals which differ only by an invertible ideal is the same, we now only have to blow up the union of the 56 strict transforms. For the proof of Proposition 6.5 
it now is sufficient to show that the blowup of $Y^{\prime}$ along the union of two of the 56 strict transforms is smooth. Actually the strict transforms are smooth, and the local analytical behavior in an intersection point is the same as $z_{1}=z_{2}$ and $z_{3}=z_{4}$ in $\mathbb{C}^{5}$. This shows that the blowup remains smooth and proves Proposition 6.5

Finally we compare the model $\tilde{Y}$ with $\bar{M}_{0,8}$. Recall that $M_{0,8}$ is the moduli space of 8 ordered points on a projective line and $\bar{M}_{0,8}$ is the Mumford compactification by marked stable curves. It is known $\mathrm{Ka}$ (see also $\mathrm{AL}$ ) that this is a projective smooth variety and that there exist regular contraction maps ( $[\mathrm{Ka}$; see also $[\mathrm{AL}])$

$$
\bar{M}_{0,8} \longrightarrow Y \text {. }
$$

From the universal property of blowing up one obtains a regular map

$$
\bar{M}_{0,8} \longrightarrow \tilde{Y} \text {. }
$$

We claim that this is biholomorphic.

6.7. Theorem. The two diagrams
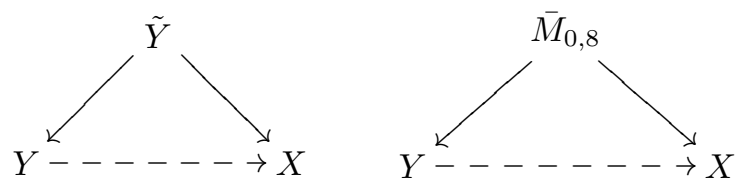

are isomorphic.

The proof rests on the following simple

6.8. Lemma. Let $V, W$ be smooth complete varieties and $f: V \rightarrow W$ a regular map which induces a biholomorphic map $V_{0} \rightarrow W_{0}$ of certain Zariski open subsets $V_{0} \subset V, W_{0} \subset W$. Assume that $V-V_{0}$ and $W-W_{0}$ are of pure codimension one and that the number of irreducible components in both cases is the same. Then $f$ is biholomorphic.

We omit the simple proof. We have to apply to $V=\bar{M}_{0,8}, V_{0}=M_{0,8}, W=\tilde{Y}$ and $W_{0}$ the complement of the inverse images of the Heegner divisors in $Y$.

Proof of Theorem 6.7. First we recall the description of the boundary components of $\bar{M}_{0,8}$. Their generic points are marked curves $\left(C, x_{1}, \ldots, x_{8}\right)$, where $C$ is the union of two $P^{1}$ with one intersection point (normal crossing). We have to choose a point $a$ on the first $P^{1}$ and $b$ on the second one, $a+b=8$. The possibilities to get a stable marked curve are $(a, b)=(6,2),(5,3),(4,4)$. In the case $(a, b)=(6,2)$ we have $28=\left(\begin{array}{l}8 \\ 2\end{array}\right)$ possibilities; in the case $(a, b)=(5,3)$, there are $56=\left(\begin{array}{l}8 \\ 3\end{array}\right)$ possibilities; in the case $(a, b)=(4,4)$, there are $35=\left(\begin{array}{l}8 \\ 4\end{array}\right) / 2$ possibilities. The denominator 2 comes from the fact that the role of the two $P^{1}$ can be interchanged. Hence we have 28,56 and 35 irreducible components. The group $S_{8}$ acts on each of the three systems transitively.

This picture can be recovered in $\tilde{Y}$. We have 28 irreducible subvarieties of codimension 1 which map to the 28 Heegner divisors in $Y$. Hence these 28 are visible already in $Y$. Then we have the 56 irreducible subvarieties of codimension 1 which come from blowing up the 56 components of the base locus. These 56 are visible in $Y^{*}$. Finally we have the 35 irreducible subvarieties of codimension 1 which come from blowing up the cusps. 
Since $\mathrm{O}\left(\mathbb{F}_{2}^{6}\right)$ permutes the three systems, this counting also shows:

6.9. Proposition. The boundary $\bar{M}_{0,8}-M_{0,8}$ is the union of $28+56+35$ irreducible subvarieties of codimension one which correspond to distributions $(6,2)$, $(5,3),(4,4)$ of eight points on two crossings $P^{1}$. Under the map $\bar{M}_{0,8} \rightarrow Y$, type $(6,2)$-components map to the 28 Heegner divisors, type $(5,3)$-components to the 56 components of the base locus and type $(4,4)$-components are contracted to the cusps.

In the next section we shall consider also the image of the boundary $\bar{M}_{0,8}-M_{0,8}$ under the map $\bar{M}_{0,8} \rightarrow X$.

\section{A COMBinatorial APPROACH}

In this section we want to give a combinatorial approach to the two different compactifications $X$ and $Y$ of the smooth hyperelliptic component. We will restrict our attention to those points in $X$ and $Y$ that do not correspond to nonsingular complete hyperelliptic curves. We shall denote by $X_{0}$ and $Y_{0}$ the open sets in $X$ and $Y$ corresponding to hyperelliptic curves.

Firstly we recall shortly the structure of $X-X_{0}$. We use the embedding of the Siegel half-plane $\mathcal{H}_{g}$ into its compact dual and we denote by $\mathcal{H}_{g}^{*}$ the union of $\mathcal{H}_{g}$ with the rational boundary components. The Satake compactification then is $\mathcal{H}_{g}^{*} / \Gamma_{g}[q]$. Details can be found for example in [Fr3], chapter II.

We restrict now to $g=3$. Recall that $X_{0}$ is the subset of all points $\mathcal{H}_{3} / \Gamma_{3}[2]$, which are represented by a (smooth) hyperelliptic Riemann surface for which $\vartheta[0]$ vanishes and that $X$ is the closure of $X_{0}$ in the Satake compactification. We recall that a point $Z \in \mathcal{H}_{3}$ is reducible if it is conjugate with respect to the action of $\Gamma_{3}$ to a point of the form

$$
\left(\begin{array}{cc}
\tau_{1} & 0 \\
0 & \tau_{2}
\end{array}\right)
$$

with $\tau_{i} \in \mathcal{H}_{g_{i}}, g_{1}+g_{2}=3$. We shall denote by $\mathcal{R}_{3}$ the set of reducible points, on which $\vartheta[0]$ vanishes. It is known that $\mathcal{R}_{3} / \Gamma_{3}[2]$ is contained in $X$ and, even more,

$$
X=X_{0} \sqcup \overline{\mathcal{R}_{3} / \Gamma_{3}[2]} \quad \text { (disjoint union). }
$$

We want to count how many reducible components appear in $X-X_{0}$. A sixtuplet of even characteristics is called azygetic if the sum of three of them results in an odd characteristic. Each reducible component of $\overline{\mathcal{R}_{3} / \Gamma_{3}[2]}$ is defined by the vanishing of 6 thetanullwerte, which belong to an azygetic sixtuplet, where one of them has to be zero. Hence we obtain:

7.1. Proposition. The boundary part $X-X_{0}$ of the hyperelliptic component $X$ consists of 56 irreducible 4-dimensional components. They correspond to sets of 6 even characteristics, one of them 0, such that all sums of three of them are odd. The component is the intersection of the corresponding zero divisors $\vartheta[m]=0$. The group $\Gamma_{3, \vartheta}$ permutes the components transitively.

Part of the boundary $X-X_{0}$ is the intersection of $X$ with the Satake boundary. Recall that

$$
\mathcal{H}_{3}^{*}-\mathcal{H}_{3}
$$

is the disjoint union of the $\Gamma_{3}$-orbits of the three standard components

$$
\left(\begin{array}{ccc}
\mathrm{i} \infty & 0 & 0 \\
0 & \mathrm{i} \infty & 0 \\
0 & 0 & \mathrm{i} \infty
\end{array}\right), \quad\left(\begin{array}{ccc}
\tau & 0 & 0 \\
0 & \mathrm{i} \infty & 0 \\
0 & 0 & \mathrm{i} \infty
\end{array}\right)\left(\tau \in \mathcal{H}_{1}\right), \quad\left(\begin{array}{cc}
\tau & 0 \\
0 & \mathrm{i} \infty
\end{array}\right)\left(\tau \in \mathcal{H}_{2}\right)
$$


We denote by $\mathcal{D}_{3}$ the part of $\mathcal{H}_{3}^{*}-\mathcal{H}_{3}$ on which $\vartheta[0]$ vanishes. We see that $\mathcal{D}_{3} / \Gamma_{3}[2]$ is contained already in the closure of the reducible locus. Hence we have the inclusions

$$
\mathcal{D}_{3} / \Gamma_{3}[2] \subset \overline{\mathcal{R}_{3} / \Gamma_{3}[2]} \subset X \subset \overline{\mathcal{H}_{3} / \Gamma_{3}[2]} .
$$

Next we want to describe the zero-dimensional boundary components of $X$. These are the points which come from the orbit of the point

$$
\left(\begin{array}{ccc}
\mathrm{i} \infty & 0 & 0 \\
0 & \mathrm{i} \infty & 0 \\
0 & 0 & \mathrm{i} \infty
\end{array}\right) .
$$

In [G] one finds the description of the zero-dimensional boundary components as intersections of 28 zero divisors $\vartheta[m]=0$. This description can be related to stars. Recall that a star is a set of 4 odd characteristics with a certain property (Definition 2.2).

7.2. Proposition. Let $S$ be a star. There are 27 even characteristics $m \neq 0$ orthogonal to at least one characteristic in $S$. The intersection of the 27 divisors $\vartheta[\mathrm{m}]=0$ is a zero-dimensional boundary component of $X$. This gives a 1-1-correspondence between the 105 stars and the 105 zero-dimensional boundary components.

We mention also how the remaining 8 even characteristics can be described in a nice way: The star $S$ spans a three-dimensional space $M$. There exists a unique even characteristic $n$ such that $M+n$ contains only even characteristics. The elements of this coset are the 8 remaining characteristics.

Now we describe the closures of the three-dimensional boundary components of $X$. They are of the type

$$
\left(\begin{array}{cc}
\tau & 0 \\
0 & \mathrm{i} \infty
\end{array}\right), \quad \tau \in \mathcal{H}_{2}
$$

In G] one finds also the description of the three-dimensional boundary components as intersections of 16 zero divisors $\vartheta[m]=0$. The description can be related to the odd characteristics.

7.3. Proposition. Let $\alpha$ be an odd characteristic. There are exactly 15 even characteristics $m \neq 0$ orthogonal to it. The corresponding divisors $\vartheta[m]=0$ intersect in a three-dimensional boundary component of $X$. This gives a 1-1-correspondence between the 28 odd characteristics and the 28 irreducible components of the boundary $\mathcal{D}_{3} / \Gamma_{3}[2]$ which are all three-dimensional.

A similar computation can be done for the one-dimensional boundary components of $\overline{\mathcal{H}_{3} / \Gamma_{3}[2]}$, knowing that in this case each component is given by the vanishing of 24 suitable thetanullwerte; cf. Gl]. In this case we get:

7.4. Proposition. Let $\alpha_{1}$ and $\alpha_{2}$ be odd characteristics such that $\alpha_{1}+\alpha_{2}$ is an even characteristic. Then there are exactly 23 even characteristics $m \neq 0$ orthogonal to $\alpha_{1}$ or to $\alpha_{2}$. The corresponding divisors $\vartheta[\mathrm{m}]=0$ intersect in a one-dimensional boundary component of $X$. This gives a 1-1-correspondence between the 210 pairs of odd characteristic of the above form and the 210 one-dimensional components of the boundary $\mathcal{D}_{3} / \Gamma_{3}[2]$.

Next we recall the structure of $Y-Y_{0}$. We have two possibilities: to use the GIT-picture or the ball picture. The GIT-picture has been explained in Koil. We 
just recall the following: Consider the map

$$
\mathbb{C}^{8} \longrightarrow\left(P^{1}\right)^{8} \longrightarrow\left(P^{1}\right)^{8} / / \mathrm{SL}(2)=Y \text {. }
$$

In section one we used the variables $X_{1}, \ldots, X_{8}$ to describe this $\mathbb{C}^{8}$. We have to consider the 28 differences $W_{i j}=X_{i}-X_{j}, 1 \leq i<j \leq 8$. The images of their zero set in $Y$ describe 28 irreducible divisors. Their union is the boundary part $Y-Y_{0}$. We now switch to the ball picture. In both pictures the space $\mathbb{F}_{2}^{6}$ occurs. In the symplectic world its elements occur as theta characteristics, and in the orthogonal world $\mathbb{F}_{2}^{6}$ occurs as a quadratic space.

In Proposition 3.3 we showed that the 28 pairs $(i, j)$ are in 1-1-correspondence to the anisotropic elements of $\mathbb{F}_{2}^{6}$, in other words to the odd characteristics. Recall that to each such element $\alpha$ we introduced an irreducible divisor (Heegner divisor) $\mathcal{B}_{\alpha}$ in $\overline{\mathcal{B} / \Gamma[1-\mathrm{i}]}$.

7.5. Proposition. In our identification $Y=\overline{\mathcal{B} / \Gamma[1-i]}$, the boundary divisor in $Y$ defined by " $W_{i j}=0$ ", cf. Proposition [3.2, corresponds to the Heegner divisor $\mathcal{B}_{\alpha}$, where $\alpha$ is the anistropic element of $\mathbb{F}_{2}^{6}$ related to $(i, j)$ as in Proposition 3.3 .

For a sequence of odd characteristics $\alpha_{1}, \ldots, \alpha_{k}$, we associate the intersection

$$
\mathcal{B}_{\alpha_{1}, \ldots, \alpha_{k}}:=\mathcal{B}_{\alpha_{1}} \cap \cdots \cap \mathcal{B}_{\alpha_{k}} .
$$

Now we want to compare the Siegel and the ball picture; i.e., we have to consider the rational map $Y--\rightarrow X$.

7.6. Proposition. Under the rational map $Y--\rightarrow X$, the 28 Heegner divisors are contracted to the 28 three-dimensional boundary components. The 210 intersections $\mathcal{B}_{\alpha_{1}, \alpha_{2}}$ with $\alpha_{1}+\alpha_{2}$ an even characteristic are contracted to the 210 one-dimensional boundary components. The 420 intersections $\mathcal{B}_{\alpha_{1}, \alpha_{2}, \alpha_{3}}$ with $\alpha_{i}+\alpha_{j}$ even characteristics $(1 \leq i<j \leq 3)$ are contracted to the 105 zero-dimensional boundary components. More precisely, the four $\mathcal{B}_{\alpha_{1}, \alpha_{2}, \alpha_{3}}$ are obtained by a star map to the same point.

The rational map is defined outside the base locus, which has been described in Proposition 6.2 as a concrete three-dimensional variety. When $B$ is a divisor in $X$ we can take its inverse image in the complement of the base locus and then take the closure $A$ in $X$. We call $A$ the pull-back of $B$. From Propositions 3.2 and 3.3 we get:

7.7. Proposition. The pull-back of the zero divisor of the $\vartheta[m], m \neq 0$, is the union of 12 Heegner divisors $\mathcal{B}_{\alpha}$, namely those such that $\alpha$ is orthogonal to $\mathrm{m}$.

Recall (Definition 2.2) that stars consist of 4 anisotropic vectors with a certain property. The union of the four $\mathcal{B}_{\alpha}$ is the star divisor $\mathcal{B}_{S}$. A direct inspection shows (one $m$ is enough):

7.8. Lemma. Let $m \neq 0$ be an even characteristic. There are five different ways to write the set of all anistropic $\alpha$, which are orthogonal to $m$, as a union of three stars. The pull-back of the zero divisor of $\vartheta[\mathrm{m}]$ is the union of any of such three star divisors.

We come now to a description of the cusps. It is known that there are 35 cusps (elements of $\overline{\mathcal{B} / \Gamma[1-\mathrm{i}]}-\mathcal{B} / \Gamma[1-\mathrm{i}]$ ). In the GIT-model they correspond to the 
unstable points. From [Koi] or as an almost direct consequence of the computational Lemma 6.3, we obtain:

7.9. Lemma. The intersection of the twelve Heegner divisors, which correspond to an even nonzero characteristic (Proposition 7.7), is a cusp. This gives a bijection between the cusps and the nonzero even characteristics.

Now we will give a combinatorial description of the base locus of the rational map $Y--\rightarrow X$. We recall that it is the union of 56 linear spaces. This has been proved in Proposition 6.2 by a computer calculation (in the strong ideal-theoretic sense). Here we will give an instrinsic (set-theoretical) description of this locus in terms of Heegner divisors.

First of all we need some preliminary facts.

7.10. Lemma. Let $\alpha_{1}$ and $\alpha_{2}$ be two odd characteristics such that

$$
\alpha_{3}=\alpha_{1}+\alpha_{2}
$$

is still an odd characteristic. Then each even characteristic $m \neq 0$ is orthogonal to at least one of the $\alpha_{i}$.

The proof is easy and can be omitted.

This discussion also shows:

7.11. Corollary. There are exactly 5 even characteristics $m \neq 0$ orthogonal to $\alpha_{1}, \alpha_{2}, \alpha_{3}$ in Lemma 7.10 .

Let us consider a different configuration. We assume that $\alpha_{1}, \alpha_{2}$ are odd but $\alpha_{1}+\alpha_{2}$ is an even characteristic. In this case 23 even nonzero characteristics are orthogonal to at least one of $\alpha_{1}, \alpha_{2}$, and both together are orthogonal to 7 even characteristics. Let us take an odd $\alpha_{3}$ such that $\alpha_{1}+\alpha_{3}$ and $\alpha_{2}+\alpha_{3}$ are even characteristics. Then 27 nonzero even characteristics are orthogonal to at least one among $\alpha_{1}, \alpha_{2}, \alpha_{3}$. There is one and only one odd characteristic $\alpha_{4}$ which added to $\alpha_{1}, \alpha_{2}, \alpha_{3}$ gives even characteristics, namely $\alpha_{4}=\alpha_{1}+\alpha_{2}+\alpha_{3}$. Hence the four odd characteristics $\alpha_{1}, \alpha_{2}, \alpha_{3}, \alpha_{4}$ form a star. From this discussion follows:

7.12. Lemma. Let $\alpha_{1}, \ldots, \alpha_{k}$ be a sequence of odd characteristics such that each even characteristic is orthogonal to at least one $\alpha_{i}$. Then the sequence contains three characteristics, say $\alpha_{1}, \alpha_{2}, \alpha_{3}$, with $\alpha_{3}=\alpha_{1}+\alpha_{2}$.

For three odd characteristics $\alpha_{1}, \alpha_{2}, \alpha_{3}$ with the property $\alpha_{1}+\alpha_{2}+\alpha_{3}=0$ we want to consider the intersection $\mathcal{B}_{\alpha_{1}, \alpha_{2}, \alpha_{3}}$. One can check that it coincides already with the intersection of any two of the three Heegner divisors, i.e.,

$$
\mathcal{B}_{\alpha_{1}, \alpha_{2}, \alpha_{3}}=\mathcal{B}_{\alpha_{1}, \alpha_{2}}=\mathcal{B}_{\alpha_{1}, \alpha_{3}}=\mathcal{B}_{\alpha_{2}, \alpha_{3}}
$$

A direct computation tells us that there are exactly 56 triplets of the form $\alpha_{1}, \alpha_{2}$, $\alpha_{3}$, with $\alpha_{1}+\alpha_{2}+\alpha_{3}=0$. With this notation we have the following.

7.13. Proposition. The base locus of the rational map from $Y$ to $X$ is the set

$$
V=\bigcup_{\alpha_{1}+\alpha_{2}+\alpha_{3}=0} \mathcal{B}_{\alpha_{1}, \alpha_{2}, \alpha_{3}} .
$$

To each $\mathcal{B}_{\alpha_{1}, \alpha_{2}, \alpha_{3}}$ corresponds a linear space of Proposition 6.2 , 
The proof is an immediate consequence of the previous lemmata and of the fact that the base locus is necessarily the union of intersections of Heegner divisors.

We know from the results of the previous sections that the blowup of the base locus produces 56 divisors in $Y^{*}$ or in $\bar{M}_{0,8}$. We are interested in the image of these divisors in $X$. From Corollary 7.11, we have exactly 5 even characteristics orthogonal to $\alpha_{1}, \alpha_{2}, \alpha_{3}$. Thus the image of $\mathcal{B}_{\alpha_{1}, \alpha_{2}, \alpha_{3}}$ is defined by the vanishing of 6 thetanullwerte whose characteristics are $\Gamma_{3, \vartheta}$-conjugate to those at the beginning of the section.

Thus, according to our previous result, they define an irreducible component of $\overline{\mathcal{R}_{3} / \Gamma_{3}[2]}$. Hence we get:

7.14. Proposition. The blowup of the base locus maps on the reducible locus of the hyperelliptic modular variety. In particular, the blowup of $\mathcal{B}_{\alpha_{1}, \alpha_{2}, \alpha_{3}}$ maps onto the component defined by the vanishing of the thetanullwerte $\vartheta[m]$ with $m$ orthogonal to $\alpha_{1}, \alpha_{2}, \alpha_{3}$.

Let us conclude by considering the image of the blowup of the cusps. First of all we observe that for each even, nonzero characteristic $m$ there are 16 even characteristics $n_{1}, \ldots, n_{16}$ such that for each index $i=1, \ldots, 16, m+n_{i}=\beta_{i}$ is an odd characteristic. These sixteen odd characteristics appear in the situation that we are going to describe.

We recall from Lemma 6.3 and Proposition 7.13 that 8 linear components $\mathcal{B}_{\alpha_{1}, \alpha_{2}, \alpha_{3}}$ intersect in a cusp $m$. Each triplet is characterized by being orthogonal to the characteristic $m$. Vice versa, according to Corollary 7.11, to each triplet $\alpha_{1}, \alpha_{2}, \alpha_{3}$ we can associate, besides the characteristics $m$, four more even characteristics that are orthogonal to them. The union of all these even characteristics appearing at least once for the eight possible triplets $\alpha_{1}, \alpha_{2}, \alpha_{3}$, related to the even characteristic $m$, gives the sixteen even characteristics

$$
n_{1}, \ldots, n_{16} \text {. }
$$

In [Gl, one finds also the description of the two-dimensional components conjugate to

$$
\left(\begin{array}{ccc}
\tau_{1} & 0 & 0 \\
0 & \tau_{2} & 0 \\
0 & 0 & \mathrm{i} \infty
\end{array}\right) \quad\left(\tau_{i} \in \mathcal{H}_{1}\right)
$$

as intersections of 18 zero divisors $\vartheta[n]=0$. The description can be related to the characteristics $0, m, n_{1} \ldots, n_{16}$. In fact we have

7.15. Proposition. The blowup of the cusps maps onto two-dimensional components in the hyperelliptic modular variety. In particular, the blowup of the cusp $m$ maps onto the component defined by the vanishing of the thetanullwerte $\vartheta[n]$, $n=0, m, n_{1}, \ldots, n_{16}$ with $m+n_{i}$ odd.

We recall that in Proposition 6.4 is given the two-dimensional variety that is the inverse image of the cusps in $Y^{*}$.

\section{REFERENCES}

[AF] Allcock, D. Freitag, E.: Cubic surfaces and Borcherds products, Commentarii Math. Helv. Vol. 77, Issue 2, 270-296 (2002) MR.1915042 (2004c:14067)

[AL] Avritzer, D. Lange, H.: The moduli spaces of hyperelliptic curves and binary forms, Math. Z. 242, 615-632 (2002) MR1981190(2004c:14051) 
[Bo] Borcherds, R.: Automorphic forms with singularities on Grassmannians, Invent. Math. 132, 491-562 (1998) MR1625724 (99c:11049)

[DP] De Concini, C. Procesi, C.: A characteristic free approach to invariant theory, Adv. Math 21 330-354 (1976) MR0422314 (54:10305)

[Fr1] Freitag, E.: Some modular forms related to cubic surfaces, Kyungpook Math. J. 43, No.3, 433-462 (2003) MR2003489 (2004h:11044)

[Fr2] Freitag, E.: Comparison of different models of the moduli space of marked cubic surfaces, Proceedings of Japanese-German Seminar, Ryushi-do, edited by T. Ibukyama and W. Kohnen, 74-79 (2002)

[Fr3] Freitag, E.: Siegelsche Modulfunktionen, Grundlehren der mathematischen Wissenschaften, 254 Berlin-Heidelberg-New York: Springer-Verlag (1983) MR871067 (88b:11027)

[FS] Freitag, E. Salvati Manni, R.: Modular forms for the even unimodular lattice of signature (2,10), J. Algebraic Geom. 16, 753-791 (2007) MR.2357689 (2009b:11088)

[Gl] Glass, J. : Theta constants of genus three, Compositio Math. 40, 123-137 (1980). MR.558261 (80m:14028)

[Ho] Howe, R.: The classical groups and invariants of bilinear forms, The Mathematical Heritage of Hermann Weyl (Durham, NC, 1987), 133-166, Proc. Sympos. Pure Math. 48, Amer. Math. Soc., Providence, RI (1988) MR974333 (90e:22022)

[HMSV1] Howard, B.J. Millson, J. Snowden, A. Vakil, R.: The projective invariants of ordered points on the line, ArXiv Mathematics e-prints math.AG/0505096 (2007)

[HMSV2] Howard, B.J. Millson, J. Snowden, A. Vakil, R.: The moduli space of $n$ points on the line is cut out by simple quadrics when $n$ is not six, ArXiv Mathematics e-prints math.AG/0607372 (2007)

[Ig1] Igusa, J.: On the graded ring of theta-constants Amer. J. Math. 86, 219-246 (1964). MR0164967 (29:2258)

[Ig2] Igusa, J.: Modular forms and projective invariants Amer. J. Math. 89, 817-855 (1967). MR.0229643 (37:5217)

[Ka] Kapranov, M.M.: Chow quotients of Grassmannians I, Adv. Sov. Math. 16 (2), 29-110 (1993) MR:1237834 (95g:14053)

[Ko1] Kondo, S.: The moduli space of Enriques surfaces and Borcherds products, J. Algebraic Geometry 11, 601-627 (2002) MR1910262(2003m:14055)

[Ko2] Kondo, S.: The moduli space of 8 points on $P^{1}(\mathbb{C})$ and automorphic forms in Algebraic Geometry Contemporary Mathematics 422, 89-106 (2007), Amer. Math. Soc. MR.2296434(2008h:14027)

[Koi] Koike, K.: The projective embedding of the configuration space $X(2,8)$, Technical Reports of Mathematical Sciences, Chiba University, 16 (2000)

[MY] Matsumoto, K. Yoshida, M.: Configuration space of 8 points on the projective line and a 5-dimensional Picard modular group, Compositio Math. 86, 265-280 (1993) MR.1219628 (94c:14038)

[Mu] Mumford, D.: Tata Lectures on Theta II, Modern Birkhäuser Classics, Reprint of the 1984 edition, 2007. MR.2307768 (2007k:14087)

[Ru1] Runge, B.: On Siegel modular form, part I, J. reine angew. Math. 436, 57-85 (1993) MR.1207281 (94c:11041)

[Ru2] Runge, B.: On Siegel modular forms, part II, Nagoya Math. J. 138, 179-197 (1995) MR 1339948 (97b:11066)

[Ts] Tsuyumune, S.: Thetanullwerte on a moduli space of curves and hyperelliptic loci, Math. Zeit. 207, 539-568 (1991) MR1119956 (92j:14037)

Mathematisches Institut, University of Heidelberg, Im Neuenheimer Feld 288, D69120 Heidelberg, Germany

E-mail address: Freitag@mathi.uni-heidelberg.de

Dipartimento di Matematica, University la Sapienza, Piazzale Aldo Moro, 2, I-00185 ROMA, ITALY

E-mail address: salvati@mat.uniroma1.it 